Article

\title{
Fuzzy Parameterized Complex Multi-Fuzzy Soft Expert Set Theory and Its Application in Decision-Making
}

\author{
Yousef Al-Qudah ${ }^{1}$ (D), Mazlan Hassan ${ }^{2}\left(\right.$ D) and Nasruddin Hassan ${ }^{1, *(D)}$ \\ 1 School of Mathematical Sciences, Faculty of Science and Technology, Universiti Kebangsaan Malaysia, \\ UKM Bangi, Selangor DE 43600, Malaysia; yousef@siswa.ukm.edu.my \\ 2 Department of Management and Marketing, Faculty of Economics and Management, Universiti Putra \\ Malaysia, UPM Serdang, Selangor DE 43400, Malaysia; mazlanhs@upm.edu.my \\ * Correspondence: nas@ukm.edu.my; Tel.: +60-19-2145-750
}

Received: 7 February 2019; Accepted: 5 March 2019; Published: 9 March 2019

\begin{abstract}
Contemporary research has refined systems with complex fuzzy sets in order to improve the design and model of real-life applications. Symmetry and antisymmetry are basic characteristics of binary relations used when modeling the decision maker's preferences. A recent focus has been the analysis of a complex data set using the properties of fuzzy concept lattice and the complex soft set. We will introduce a new concept to represent the information which utilizes the time factor, called fuzzy parameterized complex multi-fuzzy soft expert set $(F P-\mathcal{C M} \mathcal{F} \mathcal{S E S})$, and investigate part of its fundamental properties. This $F P-\mathcal{C M} \mathcal{F} \mathcal{S E S}$ model allows us to validate the information provided by an expert, at a given phase of time, using the properties of complex fuzzy sets. We then construct an algorithm based on this concept by converting it from the complex state to the real state. Eventually, we implement it to a decision-making problem to demonstrate the applicability of the suggested method. A comparison among $F P-\mathcal{C} \mathcal{M F S E S}$ and other existing methods is made to expose the dominance of the suggested method. Apart from that, we also propose the weighted fuzzy parameterized complex multi-fuzzy soft expert set and investigate its application to decision-making.
\end{abstract}

Keywords: complex multi-fuzzy set; complex multi-fuzzy soft expert set; fuzzy parameterized complex multi-fuzzy soft expert set; soft expert set; decision making

\section{Introduction}

Numerous categories of uncertainties occur in almost every domain of our daily life, which calls for useful mathematic tools for handling these kinds of uncertainties. Various mathematic tools such as fuzzy set (FS) theory [1], intuitionistic fuzzy set theory [2] and multi-fuzzy set (MFS) theory [3,4] have been built and proved to be valuable in managing various types of the problems that contain uncertainties. The fuzzy Delphi approach on military drivers' performance [5] and the extended weighted aggregated sum product assessment (WASPAS) method using intuitionistic fuzzy numbers for a website evaluation [6] are examples of fuzzy hybrids used in decision-making. Unfortunately, due to the insufficiency of parameterization tools, these mentioned theories seem to face their own challenges. In order to overcome these challenges, Molodtsov [7] proposed the theory of soft sets to manage the uncertainties in parameterized form, which is independent from the challenges that have distressed the existing theoretical methods.

In recent years, the application of this theory has been implemented in many areas such as information sciences, intelligent systems, game theory, measurement theory, probability theory and the list continues. Currently, the study on soft sets is developing promptly such as fuzzy soft sets $[8,9]$, 
intuitionistic fuzzy soft sets [10,11] and multi-fuzzy soft sets [12]. Following from there, Alkhazaleh and Salleh [13] investigated the theory of soft expert sets, which later broadened to a fuzzy soft expert set theory [14], a bipolar fuzzy soft expert set [15], a multi Q-fuzzy soft expert set [16], and a neutrosophic soft expert set $[17,18]$.

Recent studies in FS and MFS focused on establishing systems utilizing complex fuzzy sets (CFS) $[19,20]$ to better design and model the real-life applications. The 'complex' part has the capability of managing the information of uncertainty and periodicity simultaneously. These models have been utilized to denote the uncertainty and periodicity features of an object together in a single set. Since then, the studies on CFS, in theories and applications, have established promptly (e.g., [21,22]). Based on [3,19], Al-Qudah and Hassan [23] introduced a hybrid model of CFS and MFS, known as the complex multi-fuzzy set (CMFS). This model seems handy in managing difficulties associated with multidimensional characterization properties. Likewise, Al-Qudah and Hassan [24] presented the concept of complex multi-fuzzy soft sets in order to assimilate the advantages of CMFS along with sufficient parameterization tools. For making these models better functional in improving decision-making results, Al-Qudah and Hassan [25] suggested the theory of the complex multi-fuzzy soft expert set (CMFSES) in order to allow the users to be familiar with all of the experts' opinion in a single model, neglecting the need for any further cumbersome operations.

Theories such as fuzzy parameterized soft sets [26], fuzzy parameterized fuzzy soft sets [27], intuitionistic fuzzy parameterized soft sets [28] and their generalizations [29-31] have been widely studied. However, these theories that we mentioned above have a shortcoming, which is their inability to represent the information which happens over a time period. To overcome this, we design a new approach of $F P-\mathcal{C M} \mathcal{F} \mathcal{S E S}$ that has the ability to handle the uncertainty data that is captured by the amplitude term and phase term of the complex numbers, simultaneously. The contributions of this study can be summarized as below:

1. Firstly, we generalize the concept of fuzzy parameterized fuzzy soft expert set to $F P-\mathcal{C} \mathcal{M} \mathcal{F} \mathcal{E} \mathcal{S}$ to include the time frame, which is presented by the phase terms and the ability to represent multi-dimensional data.

2. $F P-\mathcal{C M F S E S}$ is used to put forth an algorithm on decision-making by converting it from a complex state to a real state and subsequently provided the detailed decision steps.

3. Lastly, we introduce the weighted fuzzy parameterized complex multi-fuzzy soft expert set and examine its application to decision-making.

Section 2 denotes some fundamental definitions and theories of the associated studies. The FP-MFSES is also introduced in this section while Section 3 presents the formulation of the $F P-\mathcal{C M F S E S}$ and its operations. In Section 4, we demonstrate some operations on $F P-\mathcal{C M F} \mathcal{F} \mathcal{E S}$ together with a few propositions and theorems, while, in Section 5, an application of the corresponding theory in decision-making is discussed. In this section too, the comparison is conducted to justify the validity of the suggested approach while Section 6 is devoted to weighted fuzzy parameterized complex multi-fuzzy soft expert set based on decision-making. Finally, Section 7 is the conclusion with suggestion for further studies.

\section{Preliminaries}

In the current section, crucial theories are outlined corresponding to MFS and CMFS that are consistent with this study as stated below.

\subsection{Multi-Fuzzy Set (MFS)}

The theory of MFS [3] is a newly developed method to denote some problems that possess multidimensional characterization properties, which seems challenging to be explained in other expansions of fuzzy set theory. The notion of a MFS is defined as follows: 
Definition 1 ([3]). Let $k$ be a positive integer and $U$ be a non-empty set. An MFS $\mathcal{A}$ in $U$ is a set of ordered sequences

$$
\mathcal{A}=\left\{\left\langle x, \mu_{1}(x), \ldots, \mu_{k}(x)\right\rangle: x \in U\right\},
$$

where $\mu_{i} \in P(U)$ (for $\left.i=1,2, \ldots, k\right)$ and $P(U)$ is the set of all fuzzy subsets of $U$.

The function $\mu_{\mathcal{A}}(x)=\left(\mu_{1}(x), \ldots, \mu_{k}(x)\right)$ is known as the multi-membership function of MFS $A$, and $k$ denotes the dimension of $\mathcal{A}$. The set of all MFS of dimension $k$ in $U$ is represented by $M^{k} F S(U)$.

\subsection{Complex Multi Fuzzy Set and Complex Multi Fuzzy Soft Expert Set}

Al-Qudah and Hassan [23] proposed a CMFS for dealing with uncertainties of two-dimensional multi-fuzzy information in which it captures the amplitude terms and phase terms of the complex numbers simultaneously.

Definition 2 ([23]). Denote $(i=\sqrt{-1})$. Let $k$ be a positive integer and $U$ be a non-empty set. A CMFS $\mathcal{A}$, defined on a universe of discourse $U$, is characterised by a multi-membership function $\mu_{\mathcal{A}}(x)=\left(\mu_{\mathcal{A}}^{j}(x)\right)$ (for $j=1,2, \ldots, k$ ), which assigns to any element $x \in U$ a complex-valued grade of multi-membership functions in $\mathcal{A}$. $\mu_{\mathcal{A}}(x)$ may all lie within the unit circle in the complex plane, and are thus of the form $\mu_{\mathcal{A}}(x)=$ $\left(r_{\mathcal{A}}^{j}(x) \cdot e^{i \omega_{\mathcal{A}}^{j}(x)}\right)($ for $j=1,2, \ldots, k)$, both $r_{\mathcal{A}}^{j}(x)$ and $\omega_{\mathcal{A}}^{j}(x)$ are real-valued and $\left(r_{A}^{j}(x)\right) \in[0,1]$ (for $j=1,2, \ldots, k)$. The CMFS $\mathcal{A}$ may be denoted as the set of ordered sequence

$$
\begin{aligned}
& \mathcal{A}=\left\{\left\langle x, \mu_{\mathcal{A}}^{1}(x)=a_{1}, \mu^{2}{ }_{\mathcal{A}}(x)=a_{2}, \ldots, \mu^{k}{ }_{\mathcal{A}}(x)=a_{k}\right\rangle: x \in U\right\} \\
& =\left\{\left\langle x, r_{\mathcal{A}}^{1}(x) \cdot e^{i \omega_{\mathcal{A}}^{1}(x)}, r_{\mathcal{A}}^{2}(x) \cdot e^{i \omega_{\mathcal{A}}^{2}(x)}, \ldots, r_{\mathcal{A}}^{k}(x) \cdot e^{i \omega_{\mathcal{A}}^{k}(x)}\right\rangle: x \in U\right\},
\end{aligned}
$$

where $\mu_{\mathcal{A}}^{j}: U \rightarrow\left\{a_{j}: a_{j} \in C,\left|a_{j}\right| \leq 1\right\}$ for $j=1,2, \ldots, k$.

The function $\left(\mu_{\mathcal{A}}(x)=r_{\mathcal{A}}^{j}(x) \cdot e^{i \omega_{\mathcal{A}}^{j}(x)}\right)($ for $j=1,2, \ldots k)$ is known as the complex multi-membership function of CMFS $\mathcal{A}, k$ denotes the dimension of $\mathcal{A}$. The set of all CMFS of dimension $k$ in $U$ is represented by $C M^{k} F S(U)$ :

$$
\text { Let } \mathcal{A}=\left\{x,\left(\left(r_{\mathcal{A}}^{j}(x) \cdot e^{i \omega_{\mathcal{A}}^{j}(x)}\right)_{j \in k}\right): x \in U\right\} \text { and } \mathcal{B}=\left\{x,\left(\left(r_{\mathcal{B}}^{j}(x) \cdot e^{i \omega_{\mathcal{B}}^{j}(x)}\right)_{j \in k}\right): x \in U\right\} \text {. }
$$

Definition 3 ([23]). For every two CMFSs of dimension $k$ in X, subset, equality, union, intersection operations, and the complement operation are defined as follows:

1. $\mathcal{A} \subset \mathcal{B}$ if and only if $r_{\mathcal{A}}^{j}(x) \leq r_{\mathcal{B}}^{j}(x)$ and $\omega_{\mathcal{A}}^{j}(x) \leq \omega_{\mathcal{B}}^{j}(x)$, for all $x \in U$ and $j=1,2, \ldots, k$.

2. $\mathcal{A}=\mathcal{B}$ if and only if $r_{\mathcal{A}}^{j}(x)=r_{\mathcal{B}}^{j}(x)$ and $\omega_{\mathcal{A}}^{j}(x)=\omega_{\mathcal{B}}^{j}(x)$, for all $x \in U$ and $j=1,2, \ldots, k$.

3. $\mathcal{A} \cup \mathcal{B}=\left\{\left\langle x, r_{\mathcal{A} \cup \mathcal{B}}^{j}(x) \cdot e^{i \omega_{\mathcal{A} \cup \mathcal{B}}^{j}(x)}\right\rangle: x \in\right\}=\left\{\left\langle x, \vee\left(r_{\mathcal{A}}^{j}(x), r_{\mathcal{B}}^{j}(x)\right) \cdot e^{i \max \left[\omega_{\mathcal{A}}^{j}(x), \omega_{\mathcal{B}}^{j}(x)\right]}\right\rangle: x \in U\right\}$, for all $j=1,2, \ldots, k$.

4. $\mathcal{A} \cap \mathcal{B}=\left\{\left\langle x, r_{\mathcal{A} \cap \mathcal{B}}^{j}(x) \cdot e^{i \omega_{\mathcal{A} \cap \mathcal{B}}^{j}(x)}\right\rangle: x \in\right\}=\left\{\left\langle x, \wedge\left(r_{\mathcal{A}}^{j}(x), r_{\mathcal{B}}^{j}(x)\right) \cdot e^{i \min \left[\omega_{\mathcal{A}}^{j}(x), \omega_{\mathcal{B}}^{j}(x)\right]}\right\rangle: x \in U\right\}$, for all $j=1,2, \ldots, k$.

5. $\mathcal{A}^{c}=\left\{x, r_{\mathcal{A}^{c}}(x) \cdot e^{i \omega_{\mathcal{A}^{c}}(x)}: x \in X\right\}=\left\{x,\left[1-r_{\mathcal{A}}^{j}(x)\right] \cdot e^{i\left[2 \pi-\omega_{\mathcal{A}}^{j}(x)\right]}: x \in U\right\}$, for all $j=1,2, \ldots, k$, where $\vee$ and $\wedge$ represents the max and min operator, respectively.

Definition 4 ([25]). Let $k$ be a positive integer, $U$ be a universe of elements, E denotes a set of parameters, $X$ represents a set of experts (agents), and $O=\{1=$ agree, $0=$ disagree $\}$ a set of opinions. Let $Z=E \times X \times O$ and $\mathcal{A} \subseteq Z$. A pair $(\mathcal{F}, \mathcal{A})$ is known as a CMFSES of dimension $k\left(C M^{k} F S E S\right)$ over $U$, where $\mathcal{F}$ is a mapping given by $\mathcal{F}: \mathcal{A} \rightarrow C M^{k} F^{U}$, where $C M^{k} F^{U}$ represents the collection of all complex multi-fuzzy subsets of $U$.

The CMFSES $(\mathcal{F}, \mathcal{A})$ can be expressed as:

$$
(\mathcal{F}, \mathcal{A})=\left\{\langle e, \mathcal{F}(e)\rangle: e \in \mathcal{A}, \mathcal{F}(e) \in C M^{k} F^{U}\right\},
$$


where

$$
\mathcal{F}(e)=\left\{\left\langle x, \mu_{\mathcal{F}(e)}^{j}(x)=r_{\mathcal{F}(e)}^{j}(x) \cdot e^{i \omega_{\mathcal{F}(e)}^{j}(x)}\right\rangle: e \in \mathcal{A}, x \in U, j=1,2, \ldots, k\right\} .
$$

\subsection{Fuzzy Parameterized Multi-Fuzzy Soft Expert Set (FP-MFSES)}

We will now introduce the concept of FP-MFSES, and provide a corresponding illustrative example.

Definition 5. Let $k$ denote a positive integer and $U$ represent a universe of set, $E$ denotes a set of parameters, $I^{z}(E)$ represents all fuzzy subsets of $E, X$ represents a set of experts (agents), and $O=$ $\{1=$ agree, $0=$ disagree $\}$ is a set of opinions. Let $Z=\varphi \times X \times O$ and $\mathcal{A} \subseteq Z$, where $\varphi \subset I^{z}(E)$. A pair $(\ddot{f}, \mathcal{A})_{\varphi}$ is called an FP-MFSES of dimension $k\left(F P-M^{k} F S E S\right)$ over $U$, where $\ddot{f}$ is a mapping expressed by

$$
\ddot{f}_{\varphi}: \mathcal{A} \rightarrow M^{k} F(U)
$$

where $M^{k} F(U)$ represents the collection of all multi-fuzzy subsets of $U$.

Example 1. Suppose that a company wants to manufacture new types of products and to take some experts opinions regarding these products. Let $U=\left\{u_{1}, u_{2}\right\}$ represent a set universe consisting of two kinds of products. Suppose the parameter set $E=\left\{e_{1}, e_{2}\right\}$ i.e., two criteria are considered in order to assess the performance of these products, where $e_{1}$ represents the "quality", which consists of three levels: excellent, very good and good, while $e_{2}$ stands for the parameter "price" which also has three levels: high, medium and low, and $Y=\left\{\frac{0.2}{e_{1}}, \frac{0.7}{e_{2}}\right\}$ a fuzzy subset of $I^{z}(E)$. Let $X=\left\{x_{1}, x_{2}\right\}$ represent a set of experts who are authorized to provide their opinions corresponding to these products.

Hence, the FP-MFSES of dimension three $(\ddot{f}, \mathcal{A})_{Y}$ can be expressed as follows:

$$
\begin{aligned}
(\ddot{f}, \mathcal{A})_{Y}= & \left\{\left(\frac{0.2}{e_{1}}, x_{1}, 1\right)=\left\{\frac{(0.8,0.44,0.6)}{u_{1}}, \frac{(1,0.65,51)}{u_{2}}\right\},\left(\frac{0.2}{e_{1}}, x_{2}, 1\right)=\left\{\frac{(0.6,0.3,0.5)}{u_{1}}, \frac{(0.4,0.6,0.0 .6)}{u_{2}}\right\},\right. \\
& \left(\frac{0.7}{e_{2}}, x_{1}, 1\right)=\left\{\frac{(0.1,0.2,0.3)}{u_{1}}, \frac{(0.8,0.8,0.6)}{u_{2}}\right\},\left(\frac{0.7}{e_{2}}, x_{2}, 1\right)=\left\{\frac{(0.7,0.5,0.7)}{u_{1}}, \frac{(0.9,0.8,0.7)}{u_{2}}\right\}, \\
& \left(\frac{0.2}{e_{1}}, x_{1}, 0\right)=\left\{\frac{(0.8,0.3,0.5)}{u_{1}}, \frac{(0.8,0.7,0.62)}{u_{2}}\right\},\left(\frac{0.2}{e_{1}}, x_{2}, 0\right)=\left\{\frac{(0.6,0.5,0.44)}{u_{1}}, \frac{(0.7,0.7,0.6)}{u_{2}}\right\}, \\
& \left.\left(\frac{0.7}{e_{2}}, x_{1}, 0\right)=\left\{\frac{(0.9,0.4,0.6)}{u_{1}}, \frac{(1,0,0.9)}{u_{2}}\right\},\left(\frac{0.7}{e_{2}}, x_{2}, 0\right)=\left\{\frac{(0.3,0.5,0.0)}{u_{1}}, \frac{(0.5,0.7,0.6)}{u_{2}}\right\}\right\} .
\end{aligned}
$$

\section{Fuzzy Parameterized Complex Multi-Fuzzy Soft Expert Set}

In this current section, we propose the concept of $F P-\mathcal{C} \mathcal{M} \mathcal{F} \mathcal{S} \mathcal{S}$ and study their characterizations. Then, we give an illustrative example of it.

Definition 6. Let $k$ denote a positive integer and $U$ represent a universe of elements, E denote a set of parameters, $F^{z}(E)$ represents the set of fuzzy subsets of $E, X$ denote a set of experts, and $\mathcal{O}=\{1=$ agree, $0=$ disagree $\}$ a set of opinions. Let $\mathcal{Z}=\Upsilon \times X \times \mathcal{O}$ and $\mathcal{A} \subseteq \mathcal{Z}$ where $Y \subset F^{Z}(E)$. Then, the pair $(f, \mathcal{A})_{\Upsilon}$ is known as fuzzy parameterized complex multi-fuzzy soft expert set of dimension $k\left(F P-\mathcal{C} \mathcal{M}^{k} \mathcal{F} \mathcal{S E S}\right)$ over $U$ if and only if $f_{Y}: \mathcal{A} \rightarrow C M^{k}(U)$ is a mapping into the set of all complex multi-fuzzy sets in $U$.

The $F P-\mathcal{C} \mathcal{M}^{k} \mathcal{F} \mathcal{S E S}(f, \mathcal{A})_{Y}$ can be written as a following set of ordered pairs:

$$
(f, \mathcal{A})_{Y}=\left\{\left(\sigma=\left[\frac{\eta_{Y}(e)}{e}, x, o\right],\left\{\frac{f_{Y}(\sigma)(u)}{u}: u \in U\right\}\right): \sigma \in \mathcal{A} \subseteq Y \times X \times \mathcal{O}, e \in E, x \in X \text { and } o \in \mathcal{O}\right\},
$$

such that $\eta_{Y}(e)$ is the corresponding membership function of the fuzzy set $Y$ and $f_{Y}(\sigma)(u)=\left[\mu_{f_{Y}(\sigma)}^{j}(u)=\right.$ $\left.r_{f_{Y}(\sigma)}^{j}(u) \cdot e^{i \omega_{f_{Y}(\sigma)}^{j}(u)}\right], \forall u \in U$ and $j=1,2, \ldots, k$, where $\mu_{f_{Y}(\sigma)}^{j}(u)$ is a complex-valued grade of multi-membership function $\forall u \in U$ and $j=1,2, \ldots, k$, for the $F P-\mathcal{C} \mathcal{M}^{k} \mathcal{F} \mathcal{S E S}(f, \mathcal{A})_{Y}$. The values of $\left[\mu_{f_{Y}(\sigma)}^{j}(u)\right]$ may all lie within the unit circle in the complex plane, and are thus of the form $\left[\mu_{f_{Y}(\sigma)}^{j}(u)=\right.$ $\left.r_{f_{Y}(\sigma)}^{j}(u) \cdot e^{i \omega_{f_{Y}(\sigma)}^{j}(u)}\right]$, where $(i=\sqrt{-1})$, each of the amplitude terms $\left[r_{f_{Y}(e)}^{j}(u)\right]$ and the phase terms 
$\left[\omega_{f_{Y}(\sigma)}^{j}(u)\right]$ are both real-valued, and $\left[r_{f_{Y}(\sigma)}^{j}(u)\right] \in[0,1], \forall j \in\{1,2, . ., k\}$. The set of all FP-CMMFSES of dimension $k$ in $U$ are denoted by $F P-\mathcal{C} \mathcal{M}^{k} \mathcal{F} \mathcal{S E S}(U)$.

It follows that, letting $U$ denote a universe of elements, $E$ represent a set of parameters, and let $(f, \mathcal{A})_{Y},(g, \mathcal{B})_{\hbar},(\mathcal{L}, \mathcal{D})_{\lambda} \in P F-\mathcal{C} \mathcal{M}^{k} \mathcal{F} \mathcal{S E S}(U)$, which is defined as below:

$$
\begin{array}{r}
(f, \mathcal{A})_{Y}=\left\{\left(\sigma=\left[\frac{\eta_{Y}(e)}{e}, x, o\right],\left\{\frac{f_{Y}(\sigma)(u)}{u}: u \in U\right\}\right): \sigma \in \mathcal{A} \subseteq Y \times X \times \mathcal{O}, e \in E, x \in X \text { and } o \in \mathcal{O}\right\}, \\
(g, \mathcal{B})_{\hbar}=\left\{\left(\sigma=\left[\frac{\eta_{\hbar}(e)}{e}, x, o\right],\left\{\frac{g_{\hbar}(\sigma)(u)}{u}: u \in U\right\}\right): \sigma \in \mathcal{A} \subseteq \hbar \times X \times \mathcal{O}, e \in E, x \in X \text { and } o \in \mathcal{O}\right\}, \\
(\mathcal{L}, \mathcal{D})_{Y}=\left\{\left(\sigma=\left[\frac{\eta_{\lambda}(e)}{e}, x, o\right],\left\{\frac{\mathcal{L}_{\lambda}(\sigma)(u)}{u}: u \in U\right\}\right): \sigma \in \mathcal{A} \subseteq \lambda \times X \times \mathcal{O}, e \in E, x \in X \text { and } o \in \mathcal{O}\right\} .
\end{array}
$$

The following example aids in demonstrating this notion.

Example 2. Assume that $U=\left\{u_{1}, u_{2}\right\}$ is a universe set, $E=\left\{e_{1}, e_{2}\right\}$ is a set of attributes and $X=\left\{x_{1}, x_{2}\right\}$ be a set of experts. If $Y=\left\{\frac{0.4}{e_{1}}, \frac{0.5}{e_{2}}\right\}$ is a subset of $F^{z}(E)$, then $f_{Y}(\sigma)(u)$ is a complex multi-fuzzy soft expert set of dimension three defined as follows:

$$
\begin{aligned}
& f_{Y}\left(e_{1}, x_{1}, 1\right)(u)=\left\{\frac{\left(0.3 e^{i 2 \pi(1 / 4)}, 0.5 e^{i 2 \pi(2 / 4)}, 0.5 e^{i 2 \pi(4 / 4)}\right)}{u_{1}}, \frac{\left(0.4 e^{i 2 \pi(2 / 4)}, 0.6 e^{i 2 \pi(3 / 4)}, 0.7 e^{i 2 \pi(3 / 4)}\right)}{u_{2}}\right\}, \\
& f_{Y}\left(e_{1}, x_{2}, 1\right)(u)=\left\{\frac{\left(0.6 e^{i 2 \pi(3 / 4)}, 0.5 e^{i 2 \pi(1 / 4)}, 0.3 e^{i 2 \pi(0 / 4)}\right)}{u_{1}}, \frac{\left(0.5 e^{i 2 \pi(2 / 4)}, 0.5 e^{i 2 \pi(2 / 4)}, 0.3 e^{i 2 \pi(2 / 4)}\right)}{u_{2}}\right\}, \\
& f_{Y}\left(e_{2}, x_{1}, 1\right)(u)=\left\{\frac{\left(0.8 e^{i 2 \pi(4 / 4)}, 0.6 e^{i 2 \pi(1 / 4)}, 0.8 e^{i 2 \pi(1 / 4)}\right)}{u_{1}}, \frac{\left(1.0 e^{i 2 \pi(1 / 4)}, 0.0 e^{i 2 \pi(2 / 4)}, 0.3 e^{i 2 \pi(3 / 4)}\right)}{u_{2}}\right\}, \\
& f_{Y}\left(e_{2}, x_{2}, 1\right)(u)=\left\{\frac{\left(0.41 e^{i 2 \pi(1 / 4)}, 0.7 e^{i 2 \pi(1 / 4)}, 0.1 e^{i 2 \pi(4 / 4)}\right)}{u_{1}}, \frac{\left(0.55 e^{i 2 \pi(2 / 4)}, 0.6 e^{i 2 \pi(4 / 4)}, 0.8 e^{i 2 \pi(1 / 4)}\right)}{u_{2}}\right\}, \\
& f_{Y}\left(e_{1}, x_{1}, 0\right)(u)=\left\{\frac{\left(0.5 e^{i 2 \pi(2 / 4)}, 0.4 e^{i 2 \pi(3 / 4)}, 0.4 e^{i 2 \pi(2 / 4)}\right)}{u_{1}}, \frac{\left(0.42 e^{i 2 \pi(2 / 4)}, 0.16 e^{i 2 \pi(2 / 4)}, 0.7 e^{i 2 \pi(3 / 4)}\right)}{u_{2}}\right\}, \\
& f_{Y}\left(e_{1}, x_{2}, 0\right)(u)=\left\{\frac{\left(0.2 e^{i 2 \pi(3 / 4)}, 0.3 e^{i 2 \pi(3 / 4)}, 0.3 e^{i 2 \pi(0 / 4)}\right)}{u_{1}}, \frac{\left(0.5 e^{i 2 \pi(2 / 4)}, 0.45 e^{i 2 \pi(2 / 4)}, 0.4 e^{i 2 \pi(1 / 4)}\right)}{u_{2}}\right\}, \\
& f_{Y}\left(e_{2}, x_{1}, 0\right)(u)=\left\{\frac{\left(0.7 e^{i 2 \pi(1 / 4)}, 0.5 e^{i 2 \pi(1 / 4)}, 0.81 e^{i 2 \pi(4 / 4)}\right)}{u_{1}}, \frac{\left(1.0 e^{i 2 \pi(1 / 4)}, 0.2 e^{i 2 \pi(3 / 4)}, 0.5 e^{i 2 \pi(2 / 4)}\right)}{u_{2}}\right\}, \\
& f_{Y}\left(e_{2}, x_{2}, 0\right)(u)=\left\{\frac{\left(0.24 e^{i 2 \pi(3 / 4)}, 0.8 e^{i 2 \pi(2 / 4)}, 0.2 e^{i 2 \pi(3 / 4)}\right)}{u_{1}}, \frac{\left(0.5 e^{i 2 \pi(2 / 4)}, 0.7 e^{i 2 \pi(4 / 4)}, 0.75 e^{i 2 \pi(2 / 4)}\right)}{u_{2}}\right\} .
\end{aligned}
$$

Thus, we can view the $F P-\mathcal{C} \mathcal{M}^{k} \mathcal{F} \mathcal{S E S}(f, \mathcal{A})_{Y}$ as being comprised of the following collection of approximations:

$$
\begin{aligned}
(f, \mathcal{A})_{Y}=\{ & \left\{\left(\frac{0.4}{e_{1}}, x_{1}, 1\right), f_{Y}\left(e_{1}, x_{1}, 1\right)(u)\right\},\left\{\left(\frac{0.4}{e_{1}}, x_{2}, 1\right), f_{Y}\left(e_{1}, x_{2}, 1\right)(u)\right\}, \\
& \left\{\left(\frac{0.5}{e_{2}}, x_{1}, 1\right), f_{Y}\left(e_{2}, x_{1}, 1\right)(u)\right\},\left\{\left(\frac{0.5}{e_{2}}, x_{2}, 1\right), f_{Y}\left(e_{2}, x_{2}, 1\right)(u)\right\}, \\
& \left\{\left(\frac{0.4}{e_{1}}, x_{1}, 0\right), f_{Y}\left(e_{2}, x_{1}, 0\right)(u)\right\},\left\{\left(\frac{0.4}{e_{1}}, x_{2}, 0\right), f_{Y}\left(e_{1}, x_{2}, 0\right)(u)\right\}, \\
& \left.\left\{\left(\frac{0.5}{e_{2}}, x_{1}, 0\right), f_{Y}\left(e_{2}, x_{1}, 0\right)(u)\right\},\left\{\left(\frac{0.5}{e_{2}}, x_{2}, 0\right), f_{Y}\left(e_{2}, x_{2}, 0\right)(u)\right\}\right\} .
\end{aligned}
$$

We now introduce the ideas of the subset and equality operations on two $F P-\mathcal{C} \mathcal{M F} \mathcal{S} \mathcal{E} S$ s in the following definition.

Definition 7. Let $(f, \mathcal{A})_{Y}$ and $(g, \mathcal{B})_{\hbar}$ be two $F P-\mathcal{C} \mathcal{M}^{k} \mathcal{F} \mathcal{S E S}$ over $U$ which then yields the following:

1. $(f, \mathcal{A})_{Y}$ is a $F P-\mathcal{C} \mathcal{M}^{k} \mathcal{F} \mathcal{S E}$-subset of $(g, \mathcal{B})_{\hbar}$ if and only if the following conditions are fulfilled for $e \in E$ :

(a) $\eta_{Y}\left(\right.$ e) is a fuzzy subset of $\eta_{\hbar}(e)$.

(b) $f_{Y}(\sigma)(u)$ is a complex multi-fuzzy subset of $g_{\hbar}(\sigma)(u)$

(i.e., $r_{f_{Y}(\sigma)}^{j}(u) \leq r_{g_{\hbar}(\sigma)}^{j}(u)$ for the amplitude terms and for the phase terms $\omega_{f_{Y}(\sigma)}^{j}(u) \leq$ $\omega_{g_{\hbar}(\sigma)}^{j}(u)$, for all $\left.\sigma \in \mathcal{A}, u \in U, j=1,2, \ldots, k\right)$. 
In this case, we write $(f, \mathcal{A}) \subseteq(g, \mathcal{B})$.

2. $(f, \mathcal{A})_{Y}$ and $(g, \mathcal{B})_{\hbar}$ are $F P-\mathcal{C} \mathcal{M}^{k} \mathcal{F} \mathcal{S E}-$ equal, if $(f, \mathcal{A})_{Y}$ is a $F P-\mathcal{C} \mathcal{M}^{k} \mathcal{F} \mathcal{S E}$-subset of $(g, \mathcal{B})_{\hbar}$ and $(g, \mathcal{B})_{\hbar}$ is a FP-CM $\mathcal{M}^{k} \mathcal{F} \mathcal{S E}$-subset of $(f, \mathcal{A})_{Y}$. In other words, $(f, \mathcal{A})_{Y}=(g, \mathcal{B})_{\hbar}$ if the following conditions are fulfilled for $e \in \mathcal{A}$ :

(a) $\eta_{Y}(e)=\eta_{\hbar}(e)$.

(b) $f_{Y}(\sigma)(u)=g_{\hbar}(\sigma)(u)\left(i . e ., r_{f_{Y}(\sigma)}^{j}(u)=r_{g_{\hbar}}^{j}(\sigma)(u)\right.$ for the amplitude terms and for the phase terms $\omega_{f_{Y}(\sigma)}^{j}(u)=\omega_{g_{\hbar}(\sigma)}^{j}(u)$, for all $\left.\sigma \in \mathcal{A}, u \in U, j=1,2, \ldots, k\right)$.

Example 3. Consider Example 2 and let $Y=\left\{\frac{0.3}{e_{1}}, \frac{0.7}{e_{2}}\right\}$ be a subset of $F^{z}(E), \hbar=\left\{\frac{0.5}{e_{1}}, \frac{0.8}{e_{2}}\right\}$ be another subset over $F^{z}(E)$. Suppose that

$$
\mathcal{A}_{Y}=\left\{\left(\frac{0.3}{e_{1}}, x_{1}, 1\right),\left(\frac{0.7}{e_{2}}, x_{2}, 0\right)\right\} \text {, and } \mathcal{A}_{\hbar}=\left\{\left(\frac{0.5}{e_{1}}, x_{1}, 1\right),\left(\frac{0.8}{e_{2}}, x_{2}, 0\right),\left(\frac{0.8}{e_{2}}, x_{2}, 1\right)\right\} .
$$

Since $Y$ is a fuzzy subset of $\hbar$, apparently $\mathcal{A}_{\Upsilon} \subset \mathcal{B}_{\hbar}$. Let $(f, \mathcal{A})_{Y}$ and $(g, \mathcal{B})_{\hbar}$ be two FP-CMM $\mathcal{M}^{3} \mathcal{F} \mathcal{S} \mathcal{S} S$ defined as follows:

and

$$
\begin{aligned}
(f, \mathcal{A})_{Y}=\{ & \left\{\left(\frac{0.3}{e_{1}}, x_{1}, 1\right),\left\{\frac{\left\langle 0.5 e^{j 2 \pi(0.4)}, 0.6 e^{j 2 \pi(0.3)}, 0.7 e^{j 2 \pi(0.5)}\right\rangle}{u_{1}}, \frac{\left\langle 0.5 e^{j 2 \pi(0.5)}, 0.9 e^{j 2 \pi(0.7)}, 0.4 e^{j 2 \pi(0.8)}\right\rangle}{u_{2}}\right\}\right\}, \\
& \left.\left\{\left(\frac{0.7}{e_{2}}, x_{2}, 0\right),\left\{\frac{\left\langle 0.3 e^{j 2 \pi(0.6)}, 0.4 e^{j 2 \pi(0.5)}, 0.3 e^{j 2 \pi(0.4)}\right\rangle}{u_{1}}, \frac{\left\langle 0.3 e^{j 2 \pi(0.1)}, 0.1 e^{j 2 \pi(0.2)}, 0.2 e^{j 2 \pi(0.5)}\right\rangle}{u_{2}}\right\}\right\}\right\} .
\end{aligned}
$$

$$
\begin{gathered}
(g, \mathcal{B})_{\hbar}=\left\{\left\{\left(\frac{0.5}{e_{1}}, x_{1}, 1\right),\left\{\frac{\left\langle 0.6 e^{j 2 \pi(0.6)}, 0.8 e^{j 2 \pi(0.5)}, 1.0 e^{j 2 \pi(0.6)}\right\rangle}{u_{1}}, \frac{\left\langle 0.55 e^{j 2 \pi(0.4)}, 0.9 e^{j 2 \pi(0.7)}, 0.5 e^{j 2 \pi(0.8)}\right\rangle}{u_{1}}\right\}\right\},\right. \\
\left\{\left(\frac{0.8}{e_{2}}, x_{2}, 0\right),\left\{\frac{\left\langle 0.7 e^{j 2 \pi(0.6)}, 0.8 e^{j 2 \pi(0.8)}, 0.4 e^{j 2 \pi(0.4)}\right\rangle}{u_{2}}, \frac{\left\langle 0.44 e^{j 2 \pi(0.2)}, 0.2 e^{2 \pi(0.7)}, 0.52 e^{j 2 \pi(0.8)}\right\rangle}{u_{2}}\right\}\right\}, \\
\left.\left\{\left(\frac{0.8}{e_{2}}, x_{2}, 1\right),\left\{\frac{\left\langle 0.6 e^{j 2 \pi(0.5)}, 0.5 e^{j 2 \pi(0.5)}, 0.33 e^{j 2 \pi(0.4)}\right\rangle}{u_{1}}, \frac{\left\langle 0.3 e^{j 2 \pi(0.1)}, 0.5 e^{j 2 \pi(0.2)}, 0.4 e^{j 2 \pi(0.3)}\right\rangle}{u_{2}}\right\}\right\}\right\} .
\end{gathered}
$$

Hence, $(f, \mathcal{A})_{Y} \subseteq(g, \mathcal{B})_{\hbar}$.

Proposition 1. Let $(f, \mathcal{A})_{\curlyvee},(g, \mathcal{B})_{\hbar},(\mathcal{L}, \mathcal{D})_{\lambda} \in P F-\mathcal{C} \mathcal{M}^{k} \mathcal{F} \mathcal{S E S}(U)$. Then,

1. $(f, \mathcal{A})_{Y}=(g, \mathcal{B})_{\hbar}$ and $(g, \mathcal{B})_{\hbar}=(\mathcal{L}, \mathcal{D})_{\lambda} \Leftrightarrow(f, \mathcal{A})_{Y}=(\mathcal{L}, \mathcal{D})_{\lambda}$.

2. $\quad(f, \mathcal{A})_{Y} \subseteq(g, \mathcal{B})_{\hbar}$ and $(g, \mathcal{B})_{\hbar} \subseteq(f, \mathcal{A})_{Y} \Leftrightarrow(f, \mathcal{A})_{Y}=(g, \mathcal{B})_{\hbar}$.

3. $(f, \mathcal{A})_{Y} \subseteq(g, \mathcal{B})_{\hbar}$ and $(g, \mathcal{B})_{\hbar} \subseteq(\mathcal{L}, \mathcal{D})_{\lambda} \Longrightarrow(f, \mathcal{A})_{Y} \subseteq(\mathcal{L}, \mathcal{D})_{\lambda}$.

Proof. The properties of $\subseteq$ and $=$ trivially follow from the definitions given above.

Following from that, we now introduce the definition of the empty and absolute $F P-\mathcal{C M F} \mathcal{F} \mathcal{S}$.

Definition 8. An $F P$-C $\mathcal{M}^{k} \mathcal{F} \mathcal{S E S}(f, \mathcal{A})_{Y}$ over $U$ is said to be $Y$-empty $F P$-C $\mathcal{M}^{k} \mathcal{F} \mathcal{S E S}$, expressed by $(f, \mathcal{A})_{\tilde{\phi}_{Y}^{k}}$, if $f_{Y}(\sigma)(u)=(0,0, \ldots, 0)$, for all $\sigma \in \mathcal{A}$ and $u \in U$ (i.e., $r_{f_{Y}^{j}(\sigma)}(u)=0$ and $\omega_{f_{Y}(\sigma)}^{j}(u)=0 \pi$, for all $\sigma \in \mathcal{A}, x \in U, j=1,2, \ldots, k)$. If $\Upsilon=\phi$, then the $\Upsilon$-empty $\operatorname{PF-C\mathcal {M}} \mathcal{M}^{k} \mathcal{F} \mathcal{S E S}(f, \mathcal{A})_{\tilde{\phi}_{Y}^{k}}$ is called empty $F P-\mathcal{C} \mathcal{M}^{k} \mathcal{F} \mathcal{S E S}$, denoted by $(f, \mathcal{A})_{\tilde{\phi}^{k}}$.

Definition 9. A FP-C $\mathcal{M}^{k} \mathcal{F} \mathcal{S E S}(f, \mathcal{A})_{Y}$ over $U$ is said to be $Y$-absolute $F P-\mathcal{C} \mathcal{M}^{k} \mathcal{F} \mathcal{S E S}$, denoted by $(f, \mathcal{A})_{\tilde{U}_{Y}^{k}}$, if $f_{Y}(\sigma)(u)=(1,1, \ldots, 1)$, for all $\sigma \in \mathcal{A}$ and $u \in U$ (i.e., $r_{f_{Y}(\sigma)}^{j}(u)=1$ and $\omega_{f_{Y}(\sigma)}^{j}(u)=2 \pi$,

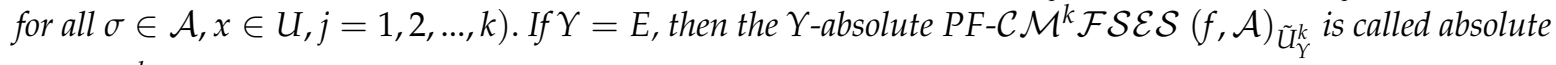
$F P-\mathcal{C} \mathcal{M}^{k} \mathcal{F} \mathcal{S E S}$, denoted by $(f, \mathcal{A})_{\tilde{U}^{k}}$.

Now, we suggest the definitions of an agree- $F P-\mathcal{C M F} \mathcal{F} \mathcal{S} S$ s and the disagree- $F P-\mathcal{C M F} \mathcal{F} \mathcal{E} S$ s. 
Definition 10. An agree-PF-C $\mathcal{M}^{k} \mathcal{F} \mathcal{S E S}(f, \mathcal{A})_{Y_{1}}$ over $U$ is a PF-C $\mathcal{M}^{k} \mathcal{F} \mathcal{S E}$ subset of $(f, \mathcal{A})_{Y}$ defined as follows:

$$
(f, \mathcal{A})_{Y_{1}}=\left\{\left(\sigma=\left[\frac{\eta_{Y}(e)}{e}, x, 1\right],\left\{\frac{f_{Y}(\sigma)(u)}{u}: u \in U\right\}\right): \sigma \in \mathcal{A} \subseteq Y \times X \times\{1\}, e \in E, x \in X \text { and } 1 \in \mathcal{O}\right\} .
$$

Definition 11. A disagree-PF-C $\mathcal{M}^{k} \mathcal{F} \mathcal{S E S}(f, \mathcal{A})_{Y_{0}}$ over $U$ is a $P F-\mathcal{C} \mathcal{M}^{k} \mathcal{F} \mathcal{S E}$ subset of $(f, \mathcal{A})_{Y}$ defined as follows:

$$
(f, \mathcal{A})_{Y_{0}}=\left\{\left(\sigma=\left[\frac{\eta_{Y}(e)}{e}, x, 0\right],\left\{\frac{f_{Y}(\sigma)(u)}{u}: u \in U\right\}\right): \sigma \in \mathcal{A} \subseteq Y \times X \times\{0\}, e \in E, x \in X \text { and } 0 \in \mathcal{O}\right\} .
$$

Example 4. Consider Example 2. The agree-PF-C $\mathcal{M}^{k} \mathcal{F} \mathcal{S E S}(f, \mathcal{A})_{Y_{1}}$ over $U$ is

$$
\begin{aligned}
(f, \mathcal{A})_{Y_{1}}=\{ & \left\{\left(\frac{0.4}{e_{1}}, x_{1}, 1\right), f_{Y}\left(e_{1}, x_{1}, 1\right)(u)\right\},\left\{\left(\frac{0.4}{e_{1}}, x_{2}, 1\right), f_{Y}\left(e_{1}, x_{2}, 1\right)(u)\right\}, \\
& \left.\left\{\left(\frac{0.5}{e_{2}}, x_{1}, 1\right), f_{Y}\left(e_{2}, x_{1}, 1\right)(u)\right\},\left\{\left(\frac{0.5}{e_{2}}, x_{2}, 1\right), f_{Y}\left(e_{2}, x_{2}, 1\right)(u)\right\}\right\},
\end{aligned}
$$

and the disagree-PF-C $\mathcal{M}^{k} \mathcal{F} \mathcal{S E S}$ is

$$
\begin{aligned}
(f, \mathcal{A})_{Y_{0}}=\{ & \left\{\left(\frac{0.4}{e_{1}}, x_{1}, 0\right), f_{Y}\left(e_{2}, x_{1}, 0\right)(u)\right\},\left\{\left(\frac{0.4}{e_{1}}, x_{2}, 0\right), f_{Y}\left(e_{1}, x_{2}, 0\right)(u)\right\}, \\
& \left.\left\{\left(\frac{0.5}{e_{2}}, x_{1}, 0\right), f_{Y}\left(e_{2}, x_{1}, 0\right)(u)\right\},\left\{\left(\frac{0.5}{e_{2}}, x_{2}, 0\right), f_{Y}\left(e_{2}, x_{2}, 0\right)(u)\right\}\right\} .
\end{aligned}
$$

\section{Basic Operations on Fuzzy Parameterized Complex Multi-Fuzzy Soft Expert Set}

In the current section, we demonstrate some fundamental theoretic operations on $F P-\mathcal{C M F} \mathcal{F} \mathcal{S} S$, which consists of the complement, union, intersection, AND and OR. We also investigate the structural features of these operations based on $F P-\mathcal{C M} \mathcal{F} \mathcal{S E S}$ s.

\subsection{Complement of FP-CMFSES}

Here, we describe the complement operation for $F P-\mathcal{C M F} \mathcal{F} \mathcal{S} S$ and provide a corresponding illustrative example and a proof of a proposed proposition.

Definition 12. Let $(f, \mathcal{A})_{Y}$ be $F P-\mathcal{C} \mathcal{M}^{k} \mathcal{F} \mathcal{S E S}$ over $U$. Then, the complement of $(f, \mathcal{A})_{Y}$ is expressed by $(f, \mathcal{A})_{Y}^{c}$ and is defined by

$$
(f, \mathcal{A})_{\Upsilon_{1}}^{c}=\left\{\left(\sigma=\left[\frac{\eta_{Y}^{c}(e)}{e}, x, o\right],\left\{\frac{f_{Y}^{c}(\sigma)(u)}{u}: u \in U\right\}\right): \sigma \in \neg \mathcal{A} \subseteq Y^{\hat{c}} \times X \times \mathcal{O}\right\},
$$

where $\eta_{Y}^{c}(e)=\hat{c}\left[\eta_{Y}(e)\right]=1-\eta_{Y}(e)$ such that $\hat{c}$ is fuzzy complement, and $f_{Y}^{c}(e)(u)=\left[\mu_{f_{Y}^{\check{c}}(e)}^{j}(u)=\right.$ $\left.r_{f_{Y}^{\check{\zeta}}(e)}^{j}(u) \cdot e^{i \omega_{\left.f_{Y}^{\check{\zeta}}(e)\right)^{(u)}}^{(u)}}=\left[1-r_{f_{Y}(e)}^{j}(u)\right] \cdot e^{i\left[2 \pi-\omega_{f_{Y}(e)}^{j}(u)\right]}\right], \forall u \in U$ and $j=1,2, \ldots, k$, where $\breve{c}$ is the complex multi-fuzzy complement.

Example 5. Consider the approximation given in Example 2, where

$$
f_{Y}\left(\frac{0.4}{e_{1}}, x_{1}, 1\right)=\left\{\frac{\left(0.3 e^{i 2 \pi(1 / 4)}, 0.5 e^{i 2 \pi(2 / 4)}, 0.5 e^{i 2 \pi(4 / 4)}\right)}{u_{1}}, \frac{\left(0.4 e^{i 2 \pi(2 / 4)}, 0.6 e^{i 2 \pi(3 / 4)}, 0.7 e^{i 2 \pi(3 / 4)}\right)}{u_{2}}\right\} .
$$

By using the above definition, we obtain the complement of the approximation, which is given by

$$
f^{\mathcal{C}}{ }_{Y}\left(\frac{0.6}{e_{1}}, x_{1}, 1\right)=\left\{\frac{\left(0.7 e^{i 2 \pi(3 / 4)}, 0.5 e^{i 2 \pi(2 / 4)}, 0.5 e^{i 2 \pi(0 / 4)}\right)}{u_{1}}, \frac{\left(0.6 e^{i 2 \pi(2 / 4)}, 0.4 e^{i 2 \pi(1 / 4)}, 0.3 e^{i 2 \pi(1 / 4)}\right)}{u_{2}}\right\} .
$$

Proposition 2. Let $(f, \mathcal{A})_{Y} \in F P-\mathcal{C} \mathcal{M}^{k} \mathcal{F} \mathcal{S E}(U)$, then $\left((f, \mathcal{A})_{Y}^{c}\right)^{c}=(f, \mathcal{A})$. 
Proof. From Definition 12, we have

$$
(f, \mathcal{A})_{Y}^{c}=\left\{\left(\sigma=\left[\frac{\eta_{Y}^{c}(e)}{e}, x, o\right],\left\{\frac{f_{Y}^{c}(\sigma)(u)}{u}: u \in U\right\}\right): \sigma \in \neg \mathcal{A} \subseteq Y^{\hat{c}} \times X \times \mathcal{O}\right\},
$$

where

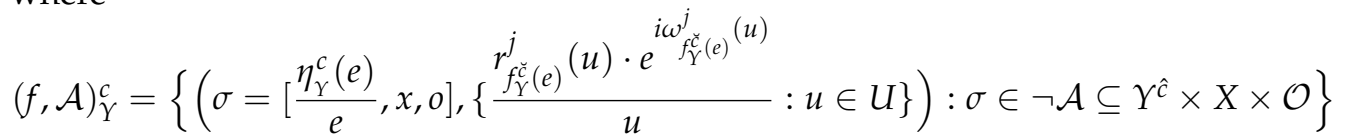

$$
\begin{aligned}
& =\left\{\left(\sigma=\left[\frac{1-\eta_{Y}(e)}{e}, x, o\right],\left\{\frac{\left[1-r_{f_{Y}(e)}^{j}(u)\right] \cdot e^{i\left[2 \pi-\omega_{f_{Y}(e)}^{j}(u)\right]}}{u}: u \in U\right\}\right): \sigma \in \neg \mathcal{A} \subseteq Y^{\hat{c}} \times X \times \mathcal{O}\right\} .
\end{aligned}
$$

Thus,

$$
\begin{aligned}
\left((f, \mathcal{A})_{Y}^{c}\right)^{c}= & \left\{\left(\sigma=\left[\frac{1-\eta_{Y}^{c}(e)}{e}, x, o\right],\left\{\frac{\left[1-r_{f_{Y}^{c}(e)}^{j}(u)\right] \cdot e^{i\left[2 \pi-\omega_{f_{Y}^{c}(e)}^{j}(u)\right]}}{u}: u \in U\right\}\right): \sigma \in \neg(\neg \mathcal{A}) \subseteq\right. \\
& \left.\left(Y^{\hat{c}}\right)^{\hat{c}} \times X \times \mathcal{O}\right\} \\
= & \left\{\left(\sigma=\left[\frac{1-\left\langle 1-\eta_{Y}(e)\right\rangle}{e}, x, o\right],\left\{\frac{\left[1-\left\langle 1-r_{f_{Y}(e)}^{j}(u)\right\rangle\right] \cdot e^{i\left[2 \pi-\left\langle 2 \pi-\omega_{f_{Y}^{j}(e)}^{j}(u)\right\rangle\right]}}{u}: u \in U\right\}\right):\right. \\
& \left.\sigma \in \neg(\neg \mathcal{A}) \subseteq\left(Y^{\hat{c}}\right)^{\hat{c}} \times X \times \mathcal{O}\right\} \\
= & \left\{\left(\sigma=\left[\frac{\eta_{Y}(e)}{e}, x, o\right],\left\{\frac{r_{f_{Y}^{j}(e)}^{j}(u) \cdot e^{i \omega_{f_{Y}(e)}^{j}(u)}}{u}: u \in U\right\}\right): \sigma \in \mathcal{A} \subseteq Y \times X \times \mathcal{O}\right\} \\
= & (f, \mathcal{A}) . \quad \square
\end{aligned}
$$

\subsection{Union and Intersection of FP-CMFSES}

In this part, we demonstrate the definitions of union and intersection operations of two $F P-\mathcal{C} \mathcal{M F S E S}$ s, along with an illustrative example and some propositions on these two operations.

Let $(f, \mathcal{A})_{Y}$ and $(g, \mathcal{B})_{\hbar}$ be two $F P-\mathcal{C} \mathcal{M}^{k} \mathcal{F S E S} S$ over a universe $U$, where $(f, \mathcal{A})_{Y}=$ $\left\{\left(\sigma=\left[\frac{\eta_{Y}(e)}{e}, x, o\right],\left\{\frac{f_{Y}(\sigma)(u)}{u}: u \in U\right\}\right): \sigma \in \mathcal{A} \subseteq Y \times X \times \mathcal{O}\right\}$, and $(g, \mathcal{B})_{\hbar}=\{(\rho=$ $\left.\left.\left[\frac{\eta_{\hbar}(e)}{e}, x, o\right],\left\{\frac{f_{\hbar}(\rho)(u)}{u}: u \in U\right\}\right): \rho \in \mathcal{B} \subseteq \hbar \times X \times \mathcal{O}\right\}$.

Definition 13. The union of $(f, \mathcal{A})_{Y}$ and $(g, \mathcal{B})_{\hbar}$, expressed by $(f, \mathcal{A})_{Y} \breve{\cup}(g, \mathcal{B})_{\hbar}$, is the $F P-\mathcal{C} \mathcal{M}^{k} \mathcal{F} \mathcal{S E S}$ $(\mathcal{T}, \mathcal{C})_{\oplus}$, where $\mathcal{C}_{\oplus}=\mathcal{A}_{Y} \cup \mathcal{B}_{\hbar}$, and $\oplus=\gamma \vec{\cup} \hbar, \vec{\cup}$ is fuzzy union, and $\forall \epsilon \in \mathcal{C}_{\oplus}, u \in U$, such that

$$
\mathcal{T}_{\oplus}(\epsilon)(u)=\left\{\begin{array}{cc}
f_{Y}(\epsilon)(u)=\left[r_{f_{Y}(\epsilon)}^{j}(u) \cdot e^{i \omega_{f_{Y}(\epsilon)}^{j}(u)}\right]_{j \in\{1,2, \ldots, k\}} & \text { if } \epsilon \in \mathcal{A}_{Y}-\mathcal{B}_{\hbar}, \\
g_{\hbar}(\epsilon)(u)=\left[r_{g_{\hbar}(\epsilon)}^{j}(u) \cdot e^{i \omega_{g_{\hbar}(\epsilon)}^{j}(u)}\right]_{j \in\{1,2, \ldots, k\}} & \text { if } \epsilon \in \mathcal{B}_{\hbar}-\mathcal{A}_{Y}, \\
f_{Y}(\epsilon)(u) \cup g_{\hbar}(\epsilon)(u)=\left[\max \left(r_{f_{Y}(\epsilon)}^{j}(u), r_{g_{\hbar}(\epsilon)}^{j}(u)\right)\right. & \\
\left.\cdot e^{i \max \left[\omega_{f_{Y}(\epsilon)}^{j}(u), \omega_{g_{\hbar}(\epsilon)}^{j}(u)\right]}\right]_{j \in\{1,2, \ldots, k\}} & \text { if } \epsilon \in \mathcal{A}_{Y} \cap \mathcal{B}_{\hbar} .
\end{array}\right.
$$

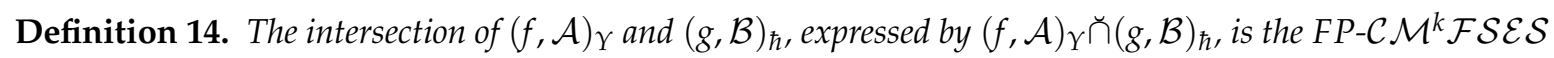
$(\mathcal{T}, \mathcal{C})_{\otimes}$, where $\mathcal{C}_{\otimes}=\mathcal{A}_{Y} \cup \mathcal{B}_{\hbar}$, and $\otimes=\Upsilon \vec{\cap} \hbar, \vec{\cap}$ is fuzzy intersection, and $\forall \epsilon \in \mathcal{C}_{\otimes}, u \in U$, such that 


$$
\mathcal{T}_{\oplus}(\epsilon)(u)=\left\{\begin{array}{cc}
f_{Y}(\epsilon)(u)=\left[r_{f_{Y}(\epsilon)}^{j}(u) \cdot e^{i \omega_{f_{Y}(\epsilon)}^{j}(u)}\right]_{j \in\{1,2, \ldots, k\}} & \text { if } \epsilon \in \mathcal{A}_{Y}-\mathcal{B}_{\hbar}, \\
g_{\hbar}(\epsilon)(u)=\left[r_{g_{\hbar}(\epsilon)}^{j}(u) \cdot e^{i \omega_{g_{\hbar}(\epsilon)}^{j}(u)}\right]_{j \in\{1,2, \ldots, k\}} & \text { if } \epsilon \in \mathcal{B}_{\hbar}-\mathcal{A}_{Y}, \\
f_{Y}(\epsilon)(u) \cap g_{\hbar}(\epsilon)(u)=\left[\min \left(r_{f_{Y}(\epsilon)}^{j}(u), r_{g_{\hbar}(\epsilon)}^{j}(u)\right)\right. & \\
\left.\cdot e^{i \min \left[\omega_{f_{Y}(\epsilon)}^{j}(u), \omega_{g_{\hbar}(\epsilon)}^{j}(u)\right]}\right]_{j \in\{1,2, \ldots, k\}} & \text { if } \epsilon \in \mathcal{A}_{Y} \cap \mathcal{B}_{\hbar} .
\end{array}\right.
$$

Example 6. Consider Example 2. Let $Y=\left\{\frac{0.6}{e_{1}}, \frac{0.4}{e_{2}}\right\}$ be a subset of $F^{z}(E)$ and $\hbar=\left\{\frac{0.5}{e_{1}}, \frac{0.8}{e_{2}}\right\}$ be another subset over $F^{z}(E)$.

$$
\mathcal{A}_{Y}=\left\{\left(\frac{0.6}{e_{1}}, x_{1}, 0\right),\left(\frac{0.4}{e_{2}}, x_{2}, 1\right)\right\}, \quad \mathcal{B}_{\hbar}=\left\{\left(\frac{0.5}{e_{1}}, x_{1}, 0\right),\left(\frac{0.8}{e_{2}}, x_{2}, 0\right),\left(\frac{0.8}{e_{2}}, x_{2}, 1\right)\right\} .
$$

Suppose $(f, \mathcal{A})_{Y}$ and $(g, \mathcal{B})_{\hbar}$ are two $F P-\mathcal{C} \mathcal{M}^{3} \mathcal{F} \mathcal{S E S}$ s over the same $U$ given by

$$
\begin{aligned}
(f, \mathcal{A})_{Y}=\{ & \left\{\left(\frac{0.6}{e^{\prime}}, x_{1}, 0\right),\left\{\frac{\left\langle 0.2 e^{j 2 \pi(0.4)}, 0.7 e^{j 2 \pi(0.4)}, 0.1 e^{j 2 \pi(0.9)}\right\rangle}{u_{1}}, \frac{\left\langle 0.8 e^{j 2 \pi(0.4)}, 0.3 e^{j 2 \pi(0.7)}, 0.3 e^{j 2 \pi(0.2)}\right\rangle}{u_{2}}\right\}\right\}, \\
& \left\{\left(\frac{0.4}{e_{2}}, x_{2}, 1\right),\left\{\frac{\left\langle 0.8 e^{j 2 \pi(0.5)}, 0.4 e^{j 2 \pi(0.5)}, 0.3 e^{j 2 \pi(0.9)}\right\rangle}{u_{1}}, \frac{\left\langle 0.3 e^{j 2 \pi(0.1)}, 0.1 e^{j 2 \pi(0.2)}, 0.9 e^{j 2 \pi(0.4)}\right.}{u_{2}}\right\}\right\},
\end{aligned}
$$

and

$$
\begin{aligned}
(g, \mathcal{B})_{\hbar}= & \left\{\left(\frac{0.5}{e_{1}}, x_{1}, 0\right),\left\{\frac{\left\langle 0.1 e^{j 2 \pi(0.6)}, 0.8 e^{j 2 \pi(0.5)}, 1.0 e^{j 2 \pi(0.6)}\right\rangle}{u_{1}}, \frac{\left\langle 0.4 e^{j 2 \pi(0.4)}, 0.9 e^{j 2 \pi(0.7)}, 0.5 e^{j 2 \pi(0.8)}\right\rangle}{u_{2}}\right\}\right\}, \\
& \left\{\left(\frac{0.8}{e_{2}}, x_{2}, 0\right),\left\{\frac{\left\langle 0.2 e^{j 2 \pi(0.5)}, 0.4 e^{j 2 \pi(0.8)}, 0.8 e^{j 2 \pi(0.4)}\right\rangle}{u_{1}}, \frac{\left\langle 0.44 e^{j 2 \pi(0.2)}, 0.2 e^{j 2 \pi(0.7)}, 0.9 e^{j 2 \pi(0.8)}\right\rangle}{u_{2}}\right\}\right\}, \\
& \left.\left\{\left(\frac{0.8}{e_{2}}, x_{2}, 1\right),\left\{\frac{\left\langle 0.8 e^{j 2 \pi(0.4)}, 0.3 e^{j 2 \pi(0.6)}, 0.4 e^{j 2 \pi(0.5)}\right\rangle}{u_{1}}, \frac{\left\langle 0.3 e^{j 2 \pi(0.1)}, 0.5 e^{j 2 \pi(0.2)}, 0.4 e^{j 2 \pi(0.3)}\right\rangle}{u_{2}}\right\}\right\}\right\} .
\end{aligned}
$$

By using Definition 13, we have

$$
\begin{aligned}
(f, \mathcal{A})_{Y} \breve{\cup}(g, \mathcal{B})_{\hbar}=\{ & \left\{\left(\frac{0.5}{e_{1}}, x_{1}, 0\right),\left\{\frac{\left\langle 0.2 e^{j 2 \pi(0.6)}, 0.8 e^{j 2 \pi(0.5)}, 1.0 e^{j 2 \pi(0.9)}\right\rangle}{u_{1}}, \frac{\left\langle 0.8 e^{j 2 \pi(0.4)}, 0.9 e^{j 2 \pi(0.7)}, 0.5 e^{j 2 \pi(0.8)}\right\rangle}{u_{2}}\right\}\right\}, \\
& \left\{\left(\frac{0.8}{e_{2}}, x_{2}, 0\right),\left\{\frac{\left\langle 0.2 e^{j 2 \pi(0.5)}, 0.4 e^{j 2 \pi(0.8)}, 0.8 e^{j 2 \pi(0.4)}\right\rangle}{u_{1}}, \frac{\left\langle 0.44 e^{j 2 \pi(0.2)}, 0.2 e^{j 2 \pi(0.7)}, 0.9 e^{j 2 \pi(0.8)}\right\rangle}{u_{2}}\right\}\right\}, \\
& \left.\left\{\left(\frac{0.8}{e_{2}}, x_{2}, 1\right),\left\{\frac{\left\langle 0.8 e^{j 2 \pi(0.55)}, 0.5 e^{j 2 \pi(0.6)}, 0.4 e^{j 2 \pi(0.9)}\right\rangle}{u_{1}}, \frac{\left\langle 0.3 e^{j 2 \pi(0.1)}, 0.5 e^{j 2 \pi(0.2)}, 0.9 e^{j 2 \pi(0.4)}\right\rangle}{u_{2}}\right\}\right\}\right\} .
\end{aligned}
$$

By using Definition 14, we have

$$
\begin{aligned}
(f, \mathcal{A})_{Y} \breve{\cap}(g, \mathcal{B})_{\hbar}=\{ & \left\{\left(\frac{0.5}{e_{1}}, x_{1}, 0\right),\left\{\frac{\left\langle 0.1 e^{j 2 \pi(0.4)}, 0.7 e^{j 2 \pi(0.4)}, 0.1 e^{j 2 \pi(0.6)}\right\rangle}{u_{1}}, \frac{\left\langle 0.4 e^{j 2 \pi(0.4)}, 0.3 e^{j 2 \pi(0.7)}, 0.3 e^{j 2 \pi(0.2)}\right\rangle}{u_{2}}\right\}\right\}, \\
& \left\{\left(\frac{0.8}{e_{2}}, x_{2}, 0\right),\left\{\frac{\left\langle 0.2 e^{j 2 \pi(0.5)}, 0.4 e^{j 2 \pi(0.8)}, 0.8 e^{j 2 \pi(0.4)}\right\rangle}{u_{1}}, \frac{\left\langle 0.44 e^{j 2 \pi(0.2)}, 0.2 e^{j 2 \pi(0.7)}, 0.9 e^{j 2 \pi(0.8)}\right\rangle}{u_{2}}\right\}\right\}, \\
& \left.\left\{\left(\frac{0.4}{e_{2}}, x_{2}, 1\right),\left\{\frac{\left\langle 0.8 e^{j 2 \pi(0.4)}, 0.3 e^{j 2 \pi(0.5)}, 0.3 e^{j 2 \pi(0.5)}\right\rangle}{u_{1}}, \frac{\left\langle 0.3 e^{j 2 \pi(0.1)}, 0.1 e^{j 2 \pi(0.2)}, 0.4 e^{j 2 \pi(0.3)}\right\rangle}{u_{2}}\right\}\right\}\right\} .
\end{aligned}
$$

The following propositions explicitly characterise the combined operations of union and intersection of $F P-\mathcal{C} \mathcal{M} \mathcal{F} \mathcal{S} \mathcal{S}$ S.

Proposition 3. Let $(f, \mathcal{A})_{Y},(g, \mathcal{B})_{\hbar}(\mathcal{L}, \mathcal{D})_{\lambda} \in F P-\mathcal{C} \mathcal{M}^{k} \mathcal{F} \mathcal{S E S}(U)$. Then,
1. $(f, \mathcal{A})_{Y} \breve{\cup}(f, \mathcal{A})_{Y}=(f, \mathcal{A})_{Y}$,
$(f, \mathcal{A})_{Y} \breve{\cap}(f, \mathcal{A})_{Y}=(f, \mathcal{A})_{Y}$.
2. $(f, \mathcal{A})_{Y} \breve{\cup}(f, \mathcal{A})_{\tilde{\phi}_{Y}^{k}}=(f, \mathcal{A})_{Y}$,
$(f, \mathcal{A})_{\Upsilon} \cap(f, \mathcal{A})_{\tilde{\phi}_{Y}^{k}}=(f, \mathcal{A})_{Y}$.
3. $\quad(f, \mathcal{A})_{Y} \breve{\cup}(f, \mathcal{A})_{\tilde{U}_{\gamma}^{k}}=(f, \mathcal{A})_{\tilde{U}_{\gamma}^{k}}$

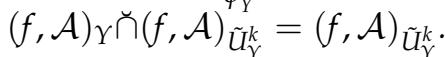

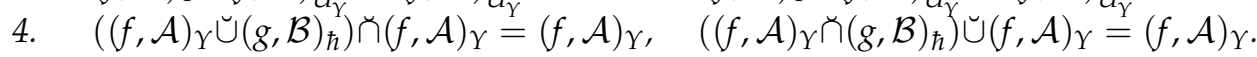

Proof. We only outline the proof of assertion 4 since the proof of assertions 1, 2 and 3 comes directly from Definitions 13 and 14.

Assume that $\left((f, \mathcal{A})_{Y} \breve{\cup}(g, \mathcal{B})_{\hbar}\right)=(R, \mathcal{P})_{\Delta}, \quad$ where $\mathcal{P}_{\Delta}=\mathcal{A}_{Y} \cup \mathcal{B}_{\hbar}, \quad$ and $\Delta=Y \vec{\cup} \hbar, \quad(S, \mathcal{W})_{\Omega}=$

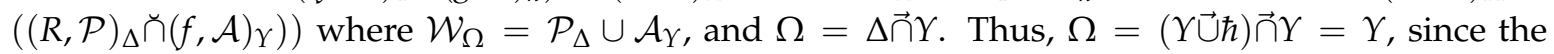
absorption property is valid for fuzzy sets. 


$$
\begin{aligned}
& \text { Let }(S, \mathcal{W})_{\Omega}=\left\{\left(\epsilon,\left\{\frac{u}{S_{\Omega}(\epsilon)(u)}: u \in U\right\}\right): \epsilon \in \mathcal{W}\right\}, \text { where } \\
& S_{\Omega}(\epsilon)(u)=\left\{\begin{array}{cc}
R(\epsilon)=\left[r_{R(\epsilon)}^{j}(u) e^{i \omega_{R(\epsilon)}^{j}(u)}\right]_{j \in\{1,2, \ldots, k\}} & \text { if } \epsilon \in \mathcal{P}_{\Delta}-\mathcal{A}_{Y}, \\
f(\epsilon)=\left[r_{f(\epsilon)}^{j}(u) e^{i \omega_{f(\epsilon)}^{j}(u)}\right]_{j \in\{1,2, \ldots, k\}} & \text { if } \epsilon \in \mathcal{A}_{Y}-\mathcal{P}_{\Delta}, \\
R(\epsilon) \cap f(\epsilon)=\left[\min \left(r_{\mathcal{R}(\epsilon)}^{j}(u), r_{f(\epsilon)}^{j}(u)\right) .\right. & \\
\left.e^{i \min \left[\omega_{R(\epsilon)}^{j}(u), \omega_{f(\epsilon)}^{j}(u)\right]}\right]_{j \in\{1,2, \ldots, k\}} & \text { if } \epsilon \in \mathcal{P}_{\Delta} \cap \mathcal{A} .
\end{array}\right.
\end{aligned}
$$

We consider the case when $\epsilon \in \mathcal{P}_{\Delta} \cap \mathcal{A}_{\lambda}$ as the other cases are trivial. Then, by using Equation (13), we have

$$
\begin{aligned}
S_{\Omega}(\epsilon)(u) & =R(\epsilon) \cap f(\epsilon) \\
& =\min \left(r_{\mathcal{R}(\epsilon)}^{j}(u), r_{f(\epsilon)}^{j}(u)\right) \cdot e^{i \min \left[\omega_{R(\epsilon)}^{j}(u), \omega_{f(\epsilon)}^{j}(u)\right]} \\
& =\min \left(r_{\left(f_{Y}(\epsilon) \vec{\cup} g_{\hbar}(\epsilon)\right)}^{j}(u), r_{f_{Y}(\epsilon)}^{j}(u)\right) \cdot e^{\left.i \min \left[\omega_{\left(f_{Y}(\epsilon) \tilde{d}_{g_{\hbar}}(\epsilon)\right)}^{j}(u), \omega_{f_{Y}(\epsilon)}^{j}(u)\right)\right]} \\
& \left.\left.=\min \left(\max \left[r_{f_{Y}(\epsilon)}^{j}(u), r_{g_{\hbar}(\epsilon)}^{j}(u)\right], r_{f_{\lambda}(\varepsilon)}^{j}(u)\right) \cdot e^{i \min \left(\operatorname { m a x } \left[\omega_{f_{Y}(\epsilon)}^{j}(u), \omega_{\delta_{\hbar}}^{j}(\epsilon)\right.\right.}(u)\right], \omega_{f_{Y}(\epsilon)}^{j}(u)\right) \\
& =\min \left(\left[r_{f_{Y}(\epsilon)}^{j}(u)\right],\left[r_{f_{Y}}^{j}(\epsilon)\right.\right. \\
& =f(\epsilon)]) \cdot e^{i \min \left(\left[\omega_{f_{Y}(\epsilon)}^{j}(u)\right],\left[\omega_{f_{Y}(\epsilon)}^{j}(u)\right]\right)} \\
& =f(\epsilon),
\end{aligned}
$$

which implies that $R(\epsilon) \sqcap f(\epsilon)=f(\epsilon)$.

Therefore, we have $\left((f, \mathcal{A})_{Y} \breve{\cup}(g, \mathcal{B})_{\hbar}\right) \breve{\cap}(f, \mathcal{A})_{Y}=(f, \mathcal{A})_{Y}$. Thus, the first part of assertion 4 is proven. Likewise, we can prove the second part of assertion 4 . This completes the proof.

\subsection{AND and $O R$ of $F P-\mathcal{C M F S E S S ~}$}

We now introduce the notion of AND and OR operations on two $F P-\mathcal{C M} \mathcal{F} \mathcal{S E S} S$ with a proposition of these two operations.

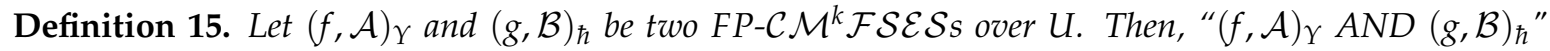
denoted by $(f, \mathcal{A})_{\Upsilon} \wedge(g, \mathcal{B})_{\hbar}$ is defined as $(f, \mathcal{A})_{\Upsilon} \wedge(g, \mathcal{B})_{\hbar}=(\mathcal{O}, \mathcal{A} \times \mathcal{B})_{\Omega}$, where $\Omega=\Upsilon \times \hbar$ and $(\mathcal{O}, \mathcal{A} \times$ $\mathcal{B})_{\Omega}=\mathcal{O}_{\Omega}(\alpha, \beta)$, such that

$$
\tilde{O}_{\Omega}(\alpha, \beta)=\left[\min \left(r_{f_{Y}(\alpha)}^{j}(u), r_{g_{\hbar}(\beta)}^{j}(u)\right) \cdot e^{i \min \left[\omega_{f_{Y}(\alpha)}^{j}(u), \omega_{g_{\hbar}(\alpha)}^{j}(u)\right]}\right],
$$

$\forall u \in U, j=1,2, \ldots, k$ and $\forall(\alpha, \beta) \in \mathcal{A}_{Y} \times \mathcal{B}_{\hbar}$.

Example 7. Consider Example 2. Let $Y=\left\{\frac{0.7}{e_{1}}, \frac{0.5}{e_{2}}\right\}$ and $\hbar=\left\{\frac{0.4}{e_{1}}, \frac{0.3}{e_{2}}\right\}$,

$$
\mathcal{A}_{Y}=\left\{\left(\frac{0.7}{e_{1}}, x_{1}, 1\right),\left(\frac{0.5}{e_{2}}, x_{2}, 0\right)\right\}, \quad \mathcal{B}_{\hbar}=\left\{\left(\frac{0.4}{e_{1}}, x_{1}, 1\right),\left(\frac{0.3}{e_{2}}, x_{2}, 1\right)\right\}
$$

Suppose $(f, \mathcal{A})_{Y}$ and $(g, \mathcal{B})_{\hbar}$ are two $F P-\mathcal{C} \mathcal{M}^{3} \mathcal{F} \mathcal{S E S}$ s over the same $U$ given by

$$
\begin{aligned}
(f, \mathcal{A})_{Y}=\{ & \left\{\left(\frac{0.6}{e_{1}}, x_{1}, 0\right),\left\{\frac{\left\langle 0.5 e^{j 2 \pi(0.6)}, 0.8 e^{j 2 \pi(0.4)}, 0.1 e^{j 2 \pi(0.9)}\right\rangle}{u_{1}}, \frac{\left\langle 0.4 e^{j 2 \pi(0.8)}, 0.5 e^{j 2 \pi(0.3)}, 0.3 e^{j 2 \pi(0.2)}\right\rangle}{u_{2}}\right\}\right\}, \\
& \left\{\left(\frac{0.4}{e_{2}}, x_{2}, 1\right),\left\{\frac{\left\langle 0.6 e^{j 2 \pi(0.45)}, 0.8 e^{j 2 \pi(0.5)}, 0.6 e^{j 2 \pi(0.9)}\right\rangle}{u_{1}}, \frac{\left\langle 0.3 e^{j 2 \pi(0.2)}, 0.1 e^{j 2 \pi(0.8)}, 0.3 e^{j 2 \pi(0.7)}\right\rangle}{u_{2}}\right\}\right\},
\end{aligned}
$$

and 


$$
\begin{aligned}
(g, \mathcal{B})_{\hbar}=\{ & \left\{\left(\frac{0.5}{e_{1}}, x_{1}, 0\right),\left\{\frac{\left\langle 0.4 e^{j 2 \pi(0.8)}, 0.5 e^{j 2 \pi(0.5)}, 0.3 e^{j 2 \pi(0.3)}\right\rangle}{u_{1}}, \frac{\left\langle 1.0 e^{j 2 \pi(0.7)}, 0.8 e^{j 2 \pi(0.2)}, 0.4 e^{j 2 \pi(0.8)}\right\rangle}{u_{2}}\right\}\right\}, \\
& \left.\left\{\left(\frac{0.8}{e_{2}}, x_{2}, 1\right),\left\{\frac{\left\langle 0.2 e^{j 2 \pi(0.4)}, 0.3 e^{j 2 \pi(0.6)}, 0.7 e^{j 2 \pi(0.5)}\right\rangle}{u_{1}}, \frac{\left\langle 0.2 e^{j 2 \pi(0.1)}, 0.8 e^{j 2 \pi(0.5)}, 0.6 e^{j 2 \pi(0.7)}\right\rangle}{u_{2}}\right\}\right\}\right\} .
\end{aligned}
$$

By using Definition 15, we have

$$
\begin{aligned}
(f, \mathcal{A})_{Y} \wedge & (g, \mathcal{B})_{\hbar}=(\mathcal{O}, \mathcal{A} \times \mathcal{B})_{\Omega}= \\
\{ & \left\{\left(\frac{0.7}{e_{1}}, x_{1}, 1\right)\left(\frac{0.4}{e_{1}}, x_{1}, 1\right),\left\{\frac{\left\langle 0.4 e^{j 2 \pi(0.6)}, 0.5 e^{j 2 \pi(0.4)}, 0.1 e^{j 2 \pi(0.3)}\right\rangle}{u_{1}}, \frac{\left\langle 0.4 e^{j 2 \pi(0.7)}, 0.5 e^{j 2 \pi(0.2)}, 0.3 e^{j 2 \pi(0.2)}\right\rangle}{u_{2}}\right\}\right\} \\
& \left\{\left(\frac{0.7}{e_{1}}, x_{1}, 1\right)\left(\frac{0.3}{e_{2}}, x_{2}, 1\right),\left\{\frac{\left\langle 0.2 e^{j 2 \pi(0.4)}, 0.3 e^{j 2 \pi(0.4)}, 0.1 e^{j 2 \pi(0.5)}\right\rangle}{u_{1}}, \frac{\left\langle 0.2 e^{j 2 \pi(0.1)}, 0.5 e^{j 2 \pi(0.3)}, 0.3 e^{j 2 \pi(0.2)}\right\rangle}{u_{2}}\right\}\right\}, \\
& \left\{\left(\frac{0.5}{e_{2}}, x_{2}, 0\right)\left(\frac{0.4}{e_{1}}, x_{1}, 1\right),\left\{\frac{\left\langle 0.4 e^{j 2 \pi(0.45)}, 0.5 e^{j 2 \pi(0.5)}, 0.3 e^{j 2 \pi(0.3)}\right\rangle}{u_{1}}, \frac{\left\langle 0.3 e^{j 2 \pi(0.2)}, 0.1 e^{j 2 \pi(0.2)}, 0.3 e^{j 2 \pi(0.7)}\right\rangle}{u_{2}}\right\}\right\}, \\
& \left.\left\{\left(\frac{0.5}{e_{2}}, x_{2}, 0\right)\left(\frac{0.3}{e_{2}}, x_{2}, 1\right),\left\{\frac{\left\langle 0.2 e^{j 2 \pi(0.4)}, 0.3 e^{j 2 \pi(0.5)}, 0.6 e^{j 2 \pi(0.5)}\right\rangle}{u_{1}}, \frac{\left\langle 0.2 e^{j 2 \pi(0.1)}, 0.1 e^{j 2 \pi(0.5)}, 0.3 e^{j 2 \pi(0.7)}\right\rangle}{u_{2}}\right\}\right\}\right\} .
\end{aligned}
$$

Definition 16. Let $(f, \mathcal{A})_{Y}$ and $(g, \mathcal{B})_{\hbar}$ be two PF-CM $\mathcal{M}^{k} \mathcal{F} \mathcal{S E S}$ s over $U$. Then, " $\left.f, \mathcal{A}\right)_{Y}$ OR $(g, \mathcal{B})_{\hbar}$ " denoted by $(f, \mathcal{A})_{\Upsilon} \vee(g, \mathcal{B})_{\hbar}$ is defined by $(f, \mathcal{A})_{\Upsilon} \vee(g, \mathcal{B})_{\hbar}=(\mathcal{O}, \mathcal{A} \times \mathcal{B})_{\Omega}$, where $\Omega=\Upsilon \times \hbar$ and $(\mathcal{O}, \mathcal{A} \times \mathcal{B})_{\Omega}=$ $\mathcal{O}_{\Omega}(\alpha, \beta)$, such that

$$
\tilde{O}_{\Omega}(\alpha, \beta)=\left[\max \left(r_{f_{Y}(\alpha)}^{j}(u), r_{g_{\hbar}(\beta)}^{j}(u)\right) \cdot e^{i \max \left[\omega_{f_{Y}(\alpha)}^{j}(u), \omega_{g_{\hbar}(\beta)}^{j}(u)\right]}\right],
$$

$\forall u \in U, j=1,2, \ldots, k$ and $\forall(\alpha, \beta) \in \mathcal{A}_{Y} \times \mathcal{B}_{\hbar}$.

Example 8. Consider Example 7. By using Definition 16, we have

$$
\begin{aligned}
(f, \mathcal{A})_{Y} \bigvee & (g, \mathcal{B})_{\hbar}=(\mathcal{O}, \mathcal{A} \times \mathcal{B})_{\Omega}= \\
\{ & \left\{\left(\frac{0.7}{e_{1}}, x_{1}, 1\right)\left(\frac{0.4}{e_{1}}, x_{1}, 1\right),\left\{\frac{\left\langle 0.5 e^{j 2 \pi(0.8)}, 0.8 e^{j 2 \pi(0.5)}, 0.3 e^{j 2 \pi(0.9)}\right\rangle}{u_{1}}, \frac{\left\langle 1.0 e^{j 2 \pi(0.8)}, 0.8 e^{j 2 \pi(0.3)}, 0.4 e^{j 2 \pi(0.8)}\right\rangle}{u_{2}}\right\}\right\}, \\
& \left\{\left(\frac{0.7}{e_{1}}, x_{1}, 1\right)\left(\frac{0.3}{e_{2}}, x_{2}, 1\right),\left\{\frac{\left\langle 0.5 e^{j 2 \pi(0.6)}, 0.8 e^{j 2 \pi(0.6)}, 0.7 e^{j 2 \pi(0.9)}\right\rangle}{u_{1}}, \frac{\left\langle 0.4 e^{j 2 \pi(0.8)}, 0.8 e^{j 2 \pi(0.5)}, 0.6 e^{j 2 \pi(0.7)}\right\rangle}{u_{2}}\right\}\right\}, \\
& \left\{\left(\frac{0.5}{e_{2}}, x_{2}, 0\right)\left(\frac{0.4}{e_{1}}, x_{1}, 1\right),\left\{\frac{\left\langle 0.6 e^{j 2 \pi(0.5)}, 0.8 e^{j 2 \pi(0.5)}, 0.6 e^{j 2 \pi(0.9)}\right\rangle}{u_{1}}, \frac{\left\langle 1.0 e^{j 2 \pi(0.8)}, 0.8 e^{j 2 \pi(0.8)}, 0.4 e^{j 2 \pi(0.8)}\right\rangle}{u_{2}}\right\}\right\}, \\
& \left.\left\{\left(\frac{0.5}{e_{2}}, x_{2}, 0\right)\left(\frac{0.3}{e_{2}}, x_{2}, 1\right),\left\{\frac{\left\langle 0.6 e^{j 2 \pi(0.45)}, 0.8 e^{j 2 \pi(0.6)}, 0.7 e^{j 2 \pi(0.9)}\right\rangle}{u_{1}}, \frac{\left\langle 0.3 e^{j 2 \pi(0.2)}, 0.8 e^{j 2 \pi(0.8)}, 0.6 e^{j 2 \pi(0.7)}\right\rangle}{u_{2}}\right\}\right\}\right\} .
\end{aligned}
$$

Proposition 4. If $(f, \mathcal{A})_{Y}$ and $\left.(g, \mathcal{B})_{\hbar}\right)$ are two $F P-\mathcal{C} \mathcal{M}^{k} \mathcal{F} \mathcal{S E S}$ s over $U$, then we have the following properties:

1. $\left((f, \mathcal{A})_{Y} \vee(g, \mathcal{B})_{\hbar}\right)^{c}=(f, \mathcal{A})_{Y}^{\mathcal{C}} \wedge(g, \mathcal{B})_{\hbar}^{c}$

2. $\left((f, \mathcal{A})_{Y} \wedge(\tilde{G}, B)\right)^{\mathcal{C}}=(f, \mathcal{A})_{Y}^{\mathcal{C}} \vee(g, \mathcal{B})_{\hbar}^{c}$.

Proof. Suppose that $(f, \mathcal{A})_{\Upsilon} \vee(g, \mathcal{B})_{\hbar}=(\mathcal{O}, \mathcal{A} \times \mathcal{B})_{\Omega}$. Then, we have $\left((f, \mathcal{A})_{\Upsilon} \vee(g, \mathcal{B})_{\hbar}\right)^{\mathcal{C}}=(\mathcal{O}, \mathcal{A} \times$ $\mathcal{B})_{\Omega}^{c}=\left(\mathcal{O}^{c}, \neg(\mathcal{A} \times \mathcal{B})\right)_{\Omega}$, where $\neg(\mathcal{A} \times \mathcal{B})_{\Omega}=\neg\left(\mathcal{A}_{Y} \vee \mathcal{B}_{\hbar}\right)_{\Omega}$. Thus, $\Omega^{\hat{c}}=(Y \vee \hbar)^{\hat{c}}=Y^{\hat{c}} \wedge \hbar^{\hat{c}}$, since the De Morgan's property is valid for fuzzy sets.

Hence,

$$
(f, \mathcal{A})_{Y}^{c} \wedge(g, \mathcal{B})_{\hbar}^{c}=\left(f^{c}, \mathcal{A}\right)_{Y} \wedge\left(g^{c}, \mathcal{B}\right)_{\hbar}=(\mathcal{J}, \neg \mathcal{A} \times \neg \mathcal{B})_{\Omega}=\left(\mathcal{J}, \neg(\mathcal{A} \times \mathcal{B})_{\Omega},\right.
$$

where $\mathcal{J}_{\Omega}(\neg \alpha, \neg \beta)=\left[\min \left(r_{f_{Y}^{c}(\neg \alpha)}^{j}(u), r_{g_{\hbar}^{c}(\neg \beta)}^{j}(u)\right) \cdot e^{i \min \left[\omega_{f_{Y}^{c}(\neg \alpha)}^{j}(u), \omega_{g_{\hbar}^{c}(\neg \alpha)}^{j}(u)\right]}\right], \forall(\neg \alpha, \neg \beta) \in(\neg \mathcal{A} \times \neg \mathcal{B})$, $j=1,2, \ldots, k$ and $u \in U$.

We take $(\neg \alpha, \neg \beta) \in(\neg \mathcal{A} \times \neg \mathcal{B})$ and $j=1,2, \ldots, k$, therefore,

$$
\begin{aligned}
\mathcal{O}_{\Omega}{ }^{c}(\neg \alpha, \neg \beta) & =r_{\mathcal{O}^{c}(\neg \alpha, \neg \beta)}^{j}(x) \cdot e^{i\left[\omega_{\mathcal{O}^{c}(\neg \alpha, \neg \beta)}^{j}(x)\right]} \\
& =\left[1-r_{\mathcal{O}(\alpha, \beta)}^{j}(x)\right] \cdot e^{i\left[2 \pi-\omega_{\mathcal{O}(\alpha, \beta)}^{j}(x)\right]} \\
& =\left[1-\max \left(r_{f_{Y}(\alpha)}^{j}(u), r_{g_{\hbar}(\beta)}^{j}(u)\right)\right] \cdot e^{i\left[2 \pi-\max \left(\omega_{f_{Y}(\alpha)}^{j}(u), \omega_{g_{\hbar}(\alpha)}^{j}(u)\right)\right]}
\end{aligned}
$$




$$
\begin{aligned}
& =\min \left(1-r_{f_{Y}(\alpha)}^{j}(u), 1-r_{g_{\hbar}(\beta)}^{j}(u)\right) \cdot e^{i \min \left(2 \pi-\omega_{f_{Y}(\alpha)}^{j}(u), 2 \pi-\omega_{g_{\hbar}(\alpha)}^{j}(u)\right)} \\
& =\min \left(r_{f_{Y}^{c}(\neg \alpha)}^{j}(u), r_{g_{\hbar}^{c}(\neg \beta)}^{j}(u)\right) \cdot e^{i \min \left[\omega_{f_{Y}^{c}(\neg \alpha)}^{j}(u), \omega_{g_{\hbar}^{c}(\neg \alpha)}^{j}(u)\right]} \\
& =\mathcal{J}_{\Omega}(\neg \alpha, \neg \beta) .
\end{aligned}
$$

Hence, $\mathcal{O}_{\Omega}{ }^{c}$ and $\mathcal{J}_{\Omega}$ are the same operator, thus the first assertion of Proposition 2 is proven. Likewise, we can prove the second assertion too.

\section{An Application of Fuzzy Parameterized Complex Multi-Fuzzy Soft Expert Set}

In this current section, we give an implementation of $F P-\mathcal{C M F} \mathcal{S E S}$ in solving a decision-making problem.

Example 9. Assume that a person wishes to purchase a travelling ticket from one of the travel agencies to travel to some countries and wants to gain opinions from some experts regarding these countries in terms of the living cost and weather. As a matter of fact, a year consists of four seasons and the living cost, and weather is different for each season. Suppose that there are three countries $u_{1}, u_{2}$ and $u_{3}$ under consideration. This set of countries is denoted by $U=\left\{u_{1}, u_{2}, u_{3}\right\}$. Suppose that the parameter set $E=\left\{e_{1}, e_{2}\right\}$ represents the factors that the traveler takes when traveling, where $e_{1}$ stands for "living cost", which includes three levels: cheap, medium and expensive; $e_{2}$ stands for "weather" which includes three cases: hot, mild and cool. The degree of importance of $e_{1}$ and $e_{2}$ are $0.4,0.6$, respectively. That is, the subset of parameters is $Y=\left\{\frac{0.4}{e_{1}}, \frac{0.6}{e_{2}}\right\}$. Let $X=\left\{x_{1}, x_{2}\right\}$ be a set of experts. Based on the input, the traveler constructed the FP-CMM $\mathcal{M}^{3} \mathcal{F} \mathcal{S} \mathcal{S}$ as follows:

$$
\begin{aligned}
& (f, \mathcal{A})_{Y}=\left\{\left\{\left(\frac{0.4}{e_{1}}, x_{1}, 1\right),\left\{\frac{\left(0.8 e^{i 2 \pi(2 / 4)}, 0.3 e^{i 2 \pi(3 / 4)}, 0.1 e^{i 2 \pi(1 / 4)}\right)}{u_{1}}, \frac{\left(0.5 e^{i 2 \pi(4 / 4)}, 0.8 e^{i 2 \pi(3 / 4)}, 0.3 e^{i 2 \pi(1 / 4)}\right)}{u_{2}},\right.\right.\right. \\
& \left.\left.\frac{\left(0.1 e^{i 2 \pi(1 / 4)}, 0.5 e^{i 2 \pi(2 / 4)}, 0.7 e^{i 2 \pi(1 / 4)}\right)}{u_{3}}\right\}\right\}, \\
& \left\{\left(\frac{0.4}{e_{1}}, x_{2}, 1\right),\left\{\frac{\left(0.6 e^{i 2 \pi(3 / 4)}, 0.22 e^{i 2 \pi(4 / 4)}, 0.7 e^{i 2 \pi(2 / 4)}\right)}{u_{1}}, \frac{\left(0.8 e^{i 2 \pi(3 / 4)}, 0.7 e^{i 2 \pi(3 / 4)}, 0.3 e^{i 2 \pi(1 / 4)}\right)}{u_{2}},\right.\right. \\
& \left.\left.\frac{\left(0.1 e^{i 2 \pi(1 / 4)}, 0.3 e^{i 2 \pi(4 / 4)}, 0.2 e^{i 2 \pi(3 / 4)}\right)}{u_{3}}\right\}\right\}, \\
& \left\{\left(\frac{0.6}{e_{2}}, x_{1}, 1\right),\left\{\frac{\left(0.1 e^{i 2 \pi(4 / 4)}, 0.5 e^{i 2 \pi(3 / 4)}, 0.2 e^{i 2 \pi(1 / 4)}\right)}{u_{1}}, \frac{\left(0.7 e^{i 2 \pi(2 / 4)}, 0.42 e^{i 2 \pi(3 / 4)}, 0.35 e^{i 2 \pi(1 / 4)}\right)}{u_{2}},\right.\right. \\
& \left.\left.\frac{\left(0.1 e^{i 2 \pi(1 / 4)}, 0.8 e^{i 2 \pi(3 / 4)}, 0.3 e^{i 2 \pi(2 / 4)}\right)}{u_{3}}\right\}\right\}, \\
& \left\{\left(\frac{0.6}{e_{2}}, x_{2}, 1\right),\left\{\frac{\left(0.2 e^{i 2 \pi(3 / 4)}, 0.5 e^{i 2 \pi(2 / 4)}, 0.8 e^{i 2 \pi(4 / 4)}\right)}{u_{1}}, \frac{\left(0.5 e^{i 2 \pi(2 / 4)}, 0.1 e^{i 2 \pi(1 / 4)}, 0.5 e^{i 2 \pi(3 / 4)}\right)}{u_{2}},\right.\right. \\
& \left.\left.\frac{\left(0.1 e^{i 2 \pi(1 / 4)}, 0.3 e^{i 2 \pi(3 / 4)}, 0.2 e^{i 2 \pi(2 / 4)}\right)}{u_{3}}\right\}\right\}, \\
& \left\{\left(\frac{0.4}{e_{1}}, x_{1}, 0\right),\left\{\frac{\left(0.2 e^{i 2 \pi(2 / 4)}, 0.7 e^{i 2 \pi(1 / 4)}, 0.9 e^{i 2 \pi(3 / 4)}\right)}{u_{1}}, \frac{\left(0.5 e^{i 2 \pi(4 / 4)}, 0.2 e^{i 2 \pi(1 / 4)}, 0.3 e^{i 2 \pi(3 / 4)}\right.}{u_{2}},\right.\right. \\
& \left.\left.\frac{\left(0.9 e^{i 2 \pi(3 / 4)}, 0.5 e^{i 2 \pi(2 / 4)}, 0.3 e^{i 2 \pi(3 / 4)}\right)}{u_{3}}\right\}\right\}, \\
& \left\{\left(\frac{0.4}{e_{1}}, x_{2}, 0\right),\left\{\frac{\left(0.35 e^{i 2 \pi(1 / 4)}, 0.6 e^{i 2 \pi(3 / 4)}, 0.1 e^{i 2 \pi(2 / 4)}\right)}{u_{1}}, \frac{\left(0.1 e^{i 2 \pi(4 / 4)}, 0.7 e^{i 2 \pi(2 / 4)}, 0.3 e^{i 2 \pi(1 / 4)}\right)}{u_{2}},\right.\right. \\
& \left.\left.\frac{\left(0.1 e^{i 2 \pi(4 / 4)}, 0.5 e^{i 2 \pi(2 / 4)}, 0.2 e^{i 2 \pi(3 / 4)}\right)}{u_{3}}\right\}\right\}, \\
& \left\{\left(\frac{0.6}{e_{2}}, x_{1}, 0\right),\left\{\frac{\left(0.2 e^{i 2 \pi(1 / 4)}, 0.5 e^{i 2 \pi(3 / 4)}, 0.8 e^{i 2 \pi(4 / 4)}\right)}{u_{1}}, \frac{\left(0.9 e^{i 2 \pi(2 / 4)}, 0.4 e^{i 2 \pi(3 / 4)}, 0.3 e^{i 2 \pi(1 / 4)}\right.}{u_{2}},\right.\right. \\
& \left.\left.\frac{\left(0.5 e^{i 2 \pi(2 / 4)}, 0.8 e^{i 2 \pi(3 / 4)}, 0.2 e^{i 2 \pi(1 / 4)}\right)}{u_{3}}\right\}\right\}, \\
& \left\{\left(\frac{0.6}{e_{2}}, x_{2}, 0\right),\left\{\frac{\left(0.8 e^{i 2 \pi(1 / 4)}, 0.5 e^{i 2 \pi(2 / 4)}, 0.2 e^{i 2 \pi(4 / 4)}\right)}{u_{1}}, \frac{\left(0.5 e^{i 2 \pi(2 / 4)}, 0.9 e^{i 2 \pi(3 / 4)}, 0.3 e^{i 2 \pi(1 / 4)}\right.}{u_{2}},\right.\right. \\
& \left.\left.\left.\frac{\left(0.1 e^{i 2 \pi(4 / 4)}, 0.5 e^{i 2 \pi(3 / 4)}, 0.2 e^{i 2 \pi(2 / 4)}\right)}{u_{3}}\right\}\right\}\right\} \text {. }
\end{aligned}
$$

In our example, the amplitude terms represent the degrees of belongingness to the living cost and weather, whereas the phase terms denote the degrees of belongingness to the phase of seasons. 
Suppose that the traveler would like to select one of the three countries based on the living cost and weather. We apply the following Algorithm 1 to this problem in order to achieve a good selection in deciding the best country in terms of the living cost and weather. We outline the steps required in this process as follows.

Algorithm 1: Using $F P-\mathcal{C} \mathcal{M}^{k} \mathcal{F} \mathcal{S E S}$.

Step 1. Construct $F P-\mathcal{C} \mathcal{M}^{k} \mathcal{F} \mathcal{S E S}(f, \mathcal{A})_{Y}$ over $U$.

Step 2. Convert the $F P-\mathcal{C} \mathcal{M}^{k} \mathcal{F} \mathcal{S E S}(f, \mathcal{A})_{Y}$ to the $F P-M^{k} F S E S(\ddot{f}, \mathcal{A})_{Y}$ by obtaining the weighted aggregation values of $\mu_{\ddot{f}_{Y}\left(\sigma_{\dot{\ell}}\right)}^{j}(u), \forall \sigma_{\dot{\ell}} \in A, \forall u_{\ell} \in U$ and $j=1,2, \ldots, k$ as in the following equation:

$$
\mu_{\ddot{f}_{Y}\left(\sigma_{\ell}\right)}^{j}\left(u_{\ell}\right)=v_{1} r_{f_{Y}\left(\sigma_{\grave{\ell}}\right)}^{j}\left(u_{\ell}\right)+v_{2}(1 / 2 \pi) \omega_{f_{Y}\left(\sigma_{\ell}\right)}^{j}\left(u_{\ell}\right),
$$

where $r_{f_{Y}\left(\sigma_{\ell}\right)}^{j}\left(u_{\ell}\right)$ and $\omega_{f_{Y}\left(\sigma_{\ell}\right)}^{j}\left(u_{\ell}\right)$ (for $\left.j=1,2, \ldots, k\right)$ are the amplitude and phase terms in the $F P-\mathcal{C} \mathcal{M}^{k} \mathcal{F} \mathcal{S E S}(f, \mathcal{A})_{Y}$, respectively. $\mu_{\ddot{f}_{Y}\left(\sigma_{\ell}\right)}^{j}\left(u_{\ell}\right)$ is the multi-membership function in the $F P-M^{k} F S E S(\ddot{f}, \mathcal{A})_{Y}$ and $v_{1}, v_{2}$ are the weights for the amplitude terms (the degrees of belongingness to the living cost and weather) and the phase terms (the degrees of belongingness to the phase of seasons), respectively, where $v_{1}$ and $v_{2} \in[0,1]$ and $v_{1}+v_{2}=1$.

Step 3. Find the values of $\mathcal{C}_{\dot{\ell} \ell}$ for agree-FP-M $M^{k} F S E S$ and disagree- $F P-M^{k} F S E S$, respectively, $\forall \sigma_{\dot{\ell}} \in \mathcal{A}$ and $\forall u_{\ell} \in U$ using

$$
\mathcal{C}_{\dot{\ell} \ell}=\frac{\sum_{j=1}^{k} \mu_{\ddot{f}_{Y}\left(\sigma_{\ell}\right)}^{j}\left(u_{\ell}\right)}{k} .
$$

Step 4. Compute the score of each element $u_{\ell} \in U$ by the following formulas:

$$
\mathcal{K}_{\ell}=\sum_{x \in X} \sum_{\dot{\ell}} \mathcal{C}_{\dot{\ell} \ell}\left(\eta_{Y}\left(e_{\dot{\ell}}\right)\right), \quad \mathcal{S}_{\ell}=\sum_{x \in X} \sum_{\dot{\ell}} \mathcal{C}_{\dot{\ell} \ell}\left(\eta_{Y}\left(e_{\dot{\ell}}\right)\right),
$$

for the agree- $F P-M^{k} F S E S$ and disagree- $F P-M^{k} F S E S$, respectively, where $\eta_{Y}\left(e_{\dot{\ell}}\right)$ is the corresponding membership function of the fuzzy set $Y$ and $X$ is the set of the experts.

Step 5. Find the value of the score $\mathcal{R}_{\ell}=\mathcal{K}_{\ell}-\mathcal{S}_{\ell}$ for each element $x_{\ell} \in U$.

Step 6. The optimal decision is any element in $s$, where $s=\max _{x_{\ell} \in U}\left\{\mathcal{R}_{\ell}\right\}$.

Now, convert the $F P-\mathcal{C} \mathcal{M}^{k} \mathcal{F} \mathcal{S E S}(f, \mathcal{A})_{Y}$ to $F P-\mathcal{M}^{k} \mathcal{F} \mathcal{S E S}(\ddot{f}, \mathcal{A})_{Y}$. To implement this step, we assume that the weight for the amplitude term is $v_{1}=0.7$ and the weight for the phase term is $v_{2}=0.3$ to obtain the weighted aggregation values of $\mu_{\ddot{f}_{Y}\left(\sigma_{\ell}\right)}^{j}\left(u_{\ell}\right), \forall \sigma_{\dot{\ell}} \in \mathcal{A}, \forall u \in U$ and $j=1,2, \ldots, k$. We calculate $\mu_{\ddot{f}_{Y}\left(\sigma_{1}\right)}^{j}\left(u_{1}\right)$, when $\sigma_{1}=\left(\frac{0.4}{e_{1}}, x_{1}, 1\right)$ and $j=1,2,3$ as shown below:

$$
\begin{aligned}
\mu_{\tilde{f}_{Y}\left(\frac{0.4}{e_{1}}, x_{1}, 1\right)}^{1}\left(u_{1}\right)= & v_{1} r_{f_{Y}\left(\frac{0.4}{e_{1}}, x_{1}, 1\right)}^{1}\left(u_{1}\right)+v_{2}(1 / 2 \pi) \omega_{f_{Y}\left(\frac{0.4}{e_{1}}, x_{1}, 1\right)}^{1}\left(u_{1}\right) \\
& =(0.7)(0.8)+(0.3)(1 / 2 \pi)(2 \pi)(2 / 4) \\
& =0.71 \\
\mu_{f_{Y}\left(\frac{0.4}{e_{1}}, x_{1}, 1\right)}^{2}\left(u_{2}\right)= & v_{1} r_{f_{Y}\left(\frac{0.4}{e_{1}}, x_{1}, 1\right)}^{2}\left(u_{2}\right)+v_{2}(1 / 2 \pi) \omega_{f_{Y}\left(\frac{0.4}{e_{1}}, x_{1}, 1\right)}^{2}\left(u_{2}\right) \\
& =(0.7)(0.3)+(0.3)(1 / 2 \pi)(2 \pi)(3 / 4) \\
& =0.435, \\
\mu_{\tilde{f}_{Y}\left(\frac{0.4}{e_{1}}, x_{1}, 1\right)}^{3}\left(u_{3}\right)= & v_{1} r_{f_{Y}\left(\frac{0.4}{e_{1}}, x_{1}, 1\right)}^{3}\left(u_{3}\right)+v_{2}(1 / 2 \pi) \omega_{f_{Y}\left(\frac{0.4}{e_{1}}, x_{1}, 1\right)}^{3}\left(u_{3}\right) \\
= & (0.7)(0.1)+(0.3)(1 / 2 \pi)(2 \pi)(1 / 4) \\
= & 0.145 .
\end{aligned}
$$


Then, for $\sigma_{1}=\left(\frac{0.4}{e_{1}}, x_{1}, 1\right)$, the $F P-M^{k} F S E$-values are given by

$$
\left(\mu_{\ddot{f}_{Y}\left(\sigma_{1}\right)}^{1}\left(u_{1}\right), \mu_{\ddot{f}_{Y}\left(\sigma_{1}\right)}^{2}\left(u_{1}\right), \mu_{\ddot{f}_{Y}\left(\sigma_{1}\right)}^{3}\left(u_{1}\right)\right)=(0.71,0.435,0.145) .
$$

In the same way, we calculate the $F P-M^{k} F S E$-values, $\forall \sigma \in \mathcal{A}$ and $\forall u \in U$ and the results are displayed in Table 1.

Table 1. Values of $(\ddot{f}, \mathcal{A})_{Y}$.

\begin{tabular}{cccc}
\hline $\boldsymbol{U}$ & $\boldsymbol{u}_{\mathbf{1}}$ & $\boldsymbol{u}_{\mathbf{2}}$ & $\boldsymbol{u}_{\mathbf{3}}$ \\
\hline$\left(\frac{0.4}{e_{1}}, x_{1}, 1\right)$ & $(0.71,0.435,0.145)$ & $(0.65,0.785,0.285)$ & $(0.145,0.5,0.565)$ \\
$\left(\frac{0.4}{e_{1}}, x_{2}, 1\right)$ & $(0.645,0.454,0.64)$ & $(0.785,0.715,0.285)$ & $(0.145,0.51,0.365)$ \\
$\left(\frac{0.6}{e_{2}}, x_{1}, 1\right)$ & $(0.37,0.575,0.215)$ & $(0.64,0.519,0.32)$ & $(0.145,0.785,0.36)$ \\
$\left(\frac{0.6}{e_{2}}, x_{2}, 1\right)$ & $(0.365,0.5,0.86)$ & $(0.5,0.145,0.575)$ & $(0.145,0.435,0.29)$ \\
$\left(\frac{0.4}{e_{1}}, x_{1}, 0\right)$ & $(0.29,0.565,0.855)$ & $(0.65,0.215,0.435)$ & $(0.855,0.5,0.435)$ \\
$\left(\frac{0.4}{e_{1}}, x_{2}, 0\right)$ & $(0.32,0.645,0.22)$ & $(0.37,0.64,0.285)$ & $(0.37,0.5,0.365)$ \\
$\left(\frac{0.6}{e_{2}}, x_{1}, 0\right)$ & $(0.215,0.575,0.86)$ & $(0.78,0.505,0.285)$ & $(0.5,0.785,0.215)$ \\
$\left(\frac{0.6}{e_{2}}, x_{2}, 0\right)$ & $(0.635,0.575,0.44)$ & $(0.5,0.855,0.285)$ & $(0.37,0.575,0.29)$ \\
\hline
\end{tabular}

From Table 1, we can give the values of $\mathcal{C}_{\ddot{\ell} \ell^{\prime}}$ for agree- $F P-M^{k} F S E S$ and disagree- $F P-M^{k} F S E S$ (as explained in Definition 5 earlier and formulated in Step 3 of Algorithm 1) presented in Tables 2 and 3, respectively.

Table 2. Tabular representation of the agree-FP-Mk $F S E S$.

\begin{tabular}{cccc}
\hline$U$ & $e_{1}$ & $e_{2}$ & $e_{3}$ \\
\hline$\left(\frac{0.4}{e_{1}}, x_{1}\right)$ & 0.43 & 0.573 & 0.403 \\
$\left(\frac{0.4}{e_{1}}, x_{2}\right)$ & 0.580 & 0.595 & 0.34 \\
$\left(\frac{0.6}{e_{2}}, x_{1}\right)$ & 0.387 & 0.493 & 0.43 \\
$\left(\frac{0.6}{e_{2}}, x_{2}\right)$ & 0.575 & 0.407 & 0.29 \\
\hline $\mathcal{K}_{\ell}=\sum_{x \in X} \sum_{\dot{\ell}} \mathcal{C}_{\ell \ell}\left(\eta_{Y}\left(e_{\ell}\right)\right)$ & $\mathcal{K}_{1}=0.981$ & $\mathcal{K}_{2}=1.007$ & $\mathcal{K}_{3}=0.729$ \\
\hline
\end{tabular}

Table 3. Tabular representation of the disagree-FP-MkFSES.

\begin{tabular}{cccc}
\hline$U$ & $e_{1}$ & $e_{2}$ & $e_{3}$ \\
\hline$\left(\frac{0.4}{e_{1}}, x_{1}\right)$ & 0.57 & 0.433 & 0.597 \\
$\left(\frac{0.4}{e_{1}}, x_{2}\right)$ & 0.395 & 0.432 & 0.412 \\
$\left(\frac{0.6}{e_{2}}, x_{1}\right)$ & 0.55 & 0.523 & 0.5 \\
$\left(\frac{0.6}{e_{2}}, x_{2}\right)$ & 0.55 & 0.547 & 0.412 \\
\hline $\mathcal{S}_{\ell}=\sum_{x \in X} \sum_{\dot{\ell}} \mathcal{C}_{\ell \ell}\left(\eta_{Y}\left(e_{\ell}\right)\right)$ & $\mathcal{S}_{1}=1.046$ & $\mathcal{S}_{2}=0.988$ & $\mathcal{S}_{3}=0.950$ \\
\hline
\end{tabular}

Let $\mathcal{K}_{\ell}$ and $\mathcal{S}_{\ell}$ represent the score of each numerical grade for the agree-FP-M $M^{k} F S E S$ and disagree- $F P-M^{k} F S E S$, respectively. These values are given in Table 4. 
Table 4. The score $\mathcal{R}_{\ell}=\mathcal{K}_{\ell}-\mathcal{S}_{\ell}$.

\begin{tabular}{cccc}
\hline $\boldsymbol{U}$ & $\mathcal{K}_{\ell}$ & $\mathcal{S}_{\ell}$ & $\mathcal{R}_{\ell}$ \\
\hline$u_{1}$ & $\mathcal{K}_{1}=0.981$ & $\mathcal{S}_{1}=1.046$ & $\mathcal{R}_{1}=-0.065$ \\
$u_{2}$ & $\mathcal{K}_{2}=1.007$ & $\mathcal{S}_{2}=0.988$ & $\mathcal{R}_{2}=0.019$ \\
$u_{3}$ & $\mathcal{K}_{3}=0.729$ & $\mathcal{S}_{3}=0.950$ & $\mathcal{R}_{3}=-0.221$ \\
\hline
\end{tabular}

Clearly, the maximum choice value is 0.019 as shown in Table 4 and so the optimal decision is to select $u_{2}$ in terms of the living cost and weather.

\section{Comparison between $F P-\mathcal{C M F S E S ~ a n d ~ t h e ~ E x i s t i n g ~ M e t h o d ~}$}

We have used the $F P-\mathcal{C M F} \mathcal{F} \mathcal{S} S$ to determine the appropriate country from the three countries to travel in terms of the living cost and weather, where its amplitude terms represent the degrees of belongingness to the living cost and weather and its phase terms represent the degrees of belongingness to the phase of seasons. In this section, we will compare our proposed $F P-\mathcal{C M} \mathcal{F} \mathcal{S E S}$ model against the existing method of a fuzzy parameterized fuzzy soft expert set (FPFSES) [31].

FPFSES is able to solve a decision-making problem using a single membership function, but it is not able to solve the decision-making problem, which involves multi-agent, multi-attribute, multi-object, multi-index and uncertainty utilizing multi-membership functions. An additional reason is its inability to deal with problems that have a periodic nature, as its structure lacks the phase term.

Consider a decision-making problem in Example 9 above. It can be seen that the FPFSES cannot describe this problem, since it is unable to represent multi-membership functions simultaneously. In addition, it is unable to represent variables in two dimensions, whereas our proposed model $F P-\mathcal{C M F} \mathcal{F} \mathcal{S} \mathcal{S}$ can completely characterize these parameters.

Thus, the proposed method has certain advantages. Firstly, this method uses the $F P-\mathcal{C M} \mathcal{F} \mathcal{S E S}$ to represent the the decision-making problem, which involves multi-agent, multi-attribute, multi-object, multi-index and uncertainty utilizing multi-membership functions. $F P-\mathcal{C} \mathcal{M} \mathcal{F} \mathcal{E} \mathcal{S}$ includes evaluation information missing in the FPFSES model, such as the time frame, which is presented by the phase terms and the ability to represent multi-dimensional data. Secondly, the $F P-\mathcal{C} \mathcal{M F S E S}$ that is used in our method has the ability to handle the uncertainty information that is captured by the amplitude terms and phase terms of the complex numbers, simultaneously. Finally, a practical formula is employed to convert the $F P-\mathcal{C} \mathcal{M F S E S ~ f r o m ~ t h e ~ c o m p l e x ~ s t a t e ~ t o ~ t h e ~ r e a l ~ s t a t e , ~ w h i c h ~ e m p l o y s ~ a ~}$ simple computational process without the need to carry out directed operations on complex numbers.

\section{Weighted of Fuzzy Parameterized Complex Multi-Fuzzy Soft Expert Set Based Decision-Making}

In this present segment, we present the idea for assigning relative weights to the experts by establishing a novel notion called $W F P-\mathcal{C M F S E S}$ and apply it to decision-making problems.

To begin with, we propose the concept of $W F P-\mathcal{C M F} \mathcal{S E S}$.

Definition 17. Let $k$ be a positive integer, $U$ be a universe of elements, $E$ be a set of parameters, $F^{z}(E)$ denote the set of fuzzy subsets of $E, X$ be a set of experts, and $F^{z}(X)$ denote all fuzzy subsets of $X$ and $\mathcal{O}=\{1=$ agree, $0=$ disagree $\}$ a set of opinions. Let $\mathcal{Z}=\Upsilon \times \mathcal{} \times \mathcal{O}$ and $\mathcal{A} \subseteq \mathcal{Z}$, where $Y \subset F^{z}(E)$ and $\omega \subset F^{z}(X)$. Then, the pair $(f, \mathcal{A})_{Y, \omega}$ is called a a weighted fuzzy parameterized complex multi-fuzzy soft expert set of dimension $k\left(\mathcal{W} F P-\mathcal{C} \mathcal{M}^{k} \mathcal{F} \mathcal{S E S}\right)$ over $U$ if and only if $f_{Y, \omega}: \mathcal{A} \rightarrow C M^{k}(U)$ is a mapping into the set of all complex multi-fuzzy sets in $U$.

The $\mathcal{W} F P-\mathcal{C} \mathcal{M}^{k} \mathcal{F} \mathcal{S E S}(f, \mathcal{A})_{Y, \omega}$ can be written as the following set of ordered pairs:

$$
(f, \mathcal{A})_{Y, \omega}=\left\{\left(\sigma=\left[\frac{\eta_{Y}(e)}{e}, \frac{\eta_{\omega}(x)}{x}, o\right],\left\{\frac{f_{Y, \omega}(\sigma)(u)}{u}: u \in U\right\}\right): \sigma \in \mathcal{A} \subseteq Y \times \omega \times \mathcal{O}, e \in E, x \in X \text { and } o \in \mathcal{O}\right\},
$$


such that $\eta_{Y}(e)$ and $\eta_{\omega}(x)$ are the corresponding membership functions of the fuzzy set $Y$ and $\omega$, respectively.

Obviously, a revised version of Algorithm 1 can be developed to cope with the decision-making

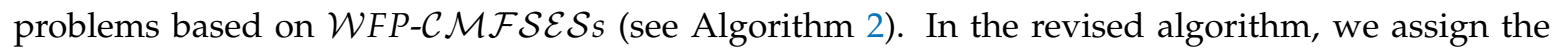
relative weight to each of the experts where the choice of the experts may not be of equal importance and compute the weighted choice values $\overline{\mathcal{R}}_{\ell}$ instead of choice values $\mathcal{R}_{\ell}$. Now, we use Algorithm 2 to select the appropriate country from the three countries of choice.

The following algorithm may be used to solve the decision-making problem in Example 9, where the data are represented by the $\mathcal{W} F P-\mathcal{C} \mathcal{M}^{k} \mathcal{F} \mathcal{S E S}(f, \mathcal{A})_{Y, \omega}$.

Algorithm 2: Using $\mathcal{W} P P-\mathcal{C} \mathcal{M}^{k} \mathcal{F} \mathcal{S E S}$.

Step 1. Construct $\mathcal{W} F P-\mathcal{C} \mathcal{M}^{k} \mathcal{F} \mathcal{S E S}(f, \mathcal{A})_{Y, \omega}$ over $U$.

Step 2. Convert the $\mathcal{W} F P-\mathcal{C} \mathcal{M}^{k} \mathcal{F} \mathcal{S} \mathcal{S}(f, \mathcal{A})_{Y, \omega}$ to the weighted fuzzy parameterized multi-fuzzy soft expert set $\left(W F P-M^{k} F S E S\right)(\ddot{f}, \mathcal{A})_{Y, \omega}$ as it was illustrated in step 2 of Algorithm 1.

Step 3. Find the values of $\mathcal{C}_{\grave{\ell} \ell}$ for agree-WFP-Mk $M^{k} S E S$ and disagree-WFP-M $M^{k} F S E S$ respectively, $\forall \sigma_{\ell} \in \mathcal{A}$ and $\forall u_{\ell} \in U$ using

$$
\mathcal{C}_{\dot{\ell} \ell}=\frac{\sum_{j=1}^{k} \mu_{\tilde{f}_{Y, \omega}\left(\sigma_{\ell}\right)}^{j}\left(u_{\ell}\right)}{k} .
$$

Step 4. Compute the score of each element $u_{\ell} \in U$ by the following formulas:

$$
\overline{\mathcal{K}}_{\ell}=\sum_{x \in X} \sum_{\dot{\ell}} \mathcal{C}_{\dot{\ell} \ell}\left(\eta_{Y}\left(e_{\dot{\ell}}\right)\right)\left(\eta_{\omega}\left(x_{\dot{\ell}}\right)\right), \quad \overline{\mathcal{S}}_{\ell}=\sum_{x \in X} \sum_{\dot{\ell}} \mathcal{C}_{\dot{\ell} \ell}\left(\eta_{Y}\left(e_{\dot{\ell}}\right)\right)\left(\eta_{\omega}\left(x_{\dot{\ell}}\right)\right)
$$

for the agree-WFP-M $M^{k} F S E S$ and disagree-WFP-Mk $M^{k} S E S$, respectively, where $\eta_{Y}\left(e_{\dot{\ell}}\right)$ and $\eta_{\omega}\left(x_{\dot{\ell}}\right)$ are the corresponding membership functions of the fuzzy set $Y$ and $\omega$, respectively.

Step 5. Find the value of the score $\overline{\mathcal{R}}_{\ell}=\overline{\mathcal{K}}_{\ell}-\overline{\mathcal{S}}_{\ell}$ for each element $u_{\ell} \in U$.

Step 6. The optimal decision is any element in $s$, where $s=\max _{u_{\ell} \in U}\left\{\overline{\mathcal{R}}_{\ell}\right\}$.

To illustrate the above idea, let us reconsider Example 9.

Example 10. Consider Example 9. Suppose that the traveler has assigned the following weights for the experts in X: for the expert " $x_{1}$ ": $\omega_{1}=0.5$, for the expert " $x_{2}$ ": $\omega_{2}=0.7$. Then, the fuzzy subset of experts is $\boldsymbol{\omega}=\left\{\frac{0.5}{x_{1}}, \frac{0.7}{x_{2}}\right\}$ and the $F P-\mathcal{C} \mathcal{M}^{k} \mathcal{F} \mathcal{S E S}(f, \mathcal{A})_{Y}$ in Example 9 is changed into a $\mathcal{W} F P-\mathcal{C} \mathcal{M}^{k} \mathcal{F} \mathcal{S E S}$ $(f, \mathcal{A})_{Y, \omega}$.

Tables 5 and 6 give the numerical grade for agree- $\mathcal{W} F P-\mathcal{M}^{k} \mathcal{F} \mathcal{S E S}$ and disagree $-\mathcal{W F P - \mathcal { M } ^ { k }} \mathcal{F} \mathcal{S E S}$.

Table 5. Tabular representation of the numerical grade for $W F P-M^{k} F S E S$.

\begin{tabular}{rccc}
\hline$U$ & $e_{1}$ & $e_{2}$ & $e_{3}$ \\
\hline$\left(\frac{0.4}{e_{1}}, \frac{0.5}{x_{1}}\right)$ & 0.43 & 0.573 & 0.403 \\
$\left(\frac{0.4}{e_{1}}, \frac{0.7}{x_{1}}\right)$ & 0.580 & 0.595 & 0.34 \\
$\left(\frac{0.6}{e_{2}}, \frac{0.5}{x_{1}}\right)$ & 0.387 & 0.493 & 0.43 \\
$\left(\frac{0.6}{e_{2}}, \frac{0.7}{x_{1}}\right)$ & 0.575 & 0.407 & 0.29 \\
\hline$\overline{\mathcal{K}}_{\ell}=\sum_{x \in X} \sum_{\dot{\ell}} \mathcal{C}_{\ell \ell}\left(\eta_{Y}\left(e_{\ell}\right)\right)\left(\eta_{\infty}\left(x_{\dot{\ell}}\right)\right)$ & $\overline{\mathcal{K}}_{1}=0.606$ & $\overline{\mathcal{K}}_{2}=0.600$ & $\overline{\mathcal{K}}_{3}=0.426$ \\
\hline
\end{tabular}


Table 6. Tabular representation of the numerical grade for disagree-WFP-M $M^{k} F S E S$.

\begin{tabular}{rccc}
\hline$U$ & $\boldsymbol{e}_{\mathbf{1}}$ & $\boldsymbol{e}_{\mathbf{2}}$ & $\boldsymbol{e}_{\mathbf{3}}$ \\
\hline$\left(\frac{0.4}{e_{1}}, \frac{0.5}{x_{1}}\right)$ & 0.57 & 0.433 & 0.597 \\
$\left(\frac{0.4}{e_{1}}, \frac{0.7}{x_{1}}\right)$ & 0.395 & 0.432 & 0.412 \\
$\left(\frac{0.6}{e_{2}}, \frac{0.5}{x_{1}}\right)$ & 0.55 & 0.523 & 0.5 \\
$\left(\frac{0.6}{e_{2}}, \frac{0.7}{x_{1}}\right)$ & 0.55 & 0.547 & 0.412 \\
\hline$\overline{\mathcal{S}}_{\ell}=\sum_{x \in X} \sum_{\dot{\ell}} \mathcal{C}_{\dot{\ell} \ell}\left(\eta_{Y}\left(e_{\dot{\ell}}\right)\right)\left(\eta_{\infty}\left(x_{\dot{\ell}}\right)\right)$ & $\overline{\mathcal{S}}_{1}=0.621$ & $\overline{\mathcal{S}}_{2}=0.594$ & $\overline{\mathcal{S}}_{3}=0.558$ \\
\hline
\end{tabular}

Now, we calculate the score $\overline{\mathcal{R}}_{\ell}=\overline{\mathcal{K}}_{\ell}-\overline{\mathcal{S}}_{\ell}$ for each element $u_{\ell} \in U$, where $\overline{\mathcal{K}}_{\ell}$ and $\overline{\mathcal{S}}_{\ell}$, represent the score of each numerical grade for the agree-WFP- $M^{k} F S E S$ and disagree-WFP- $M^{k} F S E$, respectively. The scores are tabulated in Table 7 below.

Table 7. The score $\mathcal{R}_{\ell}=\mathcal{K}_{\ell}-\mathcal{S}_{\ell}$.

\begin{tabular}{cccc}
\hline $\boldsymbol{U}$ & $\mathcal{K}_{\ell}$ & $\mathcal{S}_{\ell}$ & $\mathcal{R}_{\ell}$ \\
\hline$u_{1}$ & $\mathcal{K}_{1}=0.606$ & $\mathcal{S}_{1}=0.621$ & $\overline{\mathcal{R}}_{1}=-0.015$ \\
$u_{2}$ & $\mathcal{K}_{2}=0.600$ & $\mathcal{S}_{2}=0.594$ & $\overline{\mathcal{R}}_{2}=0.006$ \\
$u_{3}$ & $\mathcal{K}_{3}=0.426$ & $\mathcal{S}_{3}=0.558$ & $\overline{\mathcal{R}}_{3}=-0.132$ \\
\hline
\end{tabular}

From Table 7, it is clear that the weighted optimal choice value $\max _{1 \leq \ell \leq 3}\left\{\overline{\mathcal{R}}_{\ell}\right\}=\overline{\mathcal{R}}_{2}$, so the optimal decision is to select $u_{2}$. Hence, it is recommended for the traveler to choose the country $u_{2}$ as the desirable alternative.

Note that the results obtained using Algorithm 1 and Algorithm 2 are the same. However, by incorporating weights on the experts' advice may reduce the biased information given by the experts.

\section{Conclusions}

Al-Qudah and Hassan [25] introduced the idea of CMFSES as a substantial and important generalization of the soft expert set and complex multi-fuzzy set and concentrated on the utilization of CMFSES based decision-making. In this paper, we have presented the idea of $F P-\mathcal{C M} \mathcal{F} \mathcal{S E S}$ by giving an important degree to each parameter in the CMFSES's domain. In addition, we discussed some basic operations of $F P-\mathcal{C} \mathcal{M F} \mathcal{S E S}$ like equality, subset, complement, intersection, union, AND operation, and OR operation and some properties and illustrative examples were provided too. Then, we introduced an adjustable approach to decision-making using the $F P-\mathcal{C M F} \mathcal{F} \mathcal{S} \mathcal{S}$ theory and its associated algorithm constructed. This algorithm is then applied to determine the best country in terms of the living cost and weather, where its amplitude terms represent the degrees of belongingness to the living cost and weather, and its phase terms represent the degrees of belongingness to the phase of seasons. The advantage of using $F P-\mathcal{C M F} \mathcal{F E S}$ is manifested in representing information of two dimensions for one object simultaneously or objects with multi-dimensional characterization properties in one model. This type of decision-making problems cannot be solved by conventional methods such as the FPFSES [31]. A comparison of the FPFSES to $F P-\mathcal{C M} \mathcal{F} \mathcal{S E S}$ was presented and the preferability of $F P-\mathcal{C} \mathcal{M F S E S}$ was revealed. Finally, we defined the notion of weighted $F P-\mathcal{C M F} \mathcal{F} \mathcal{S}$ where experts' relative weights have been considered and applied it to solve a decision-making problem. Our preliminary study is yet to be applied to problems of many fields that contain uncertainty such as pattern recognition, image processing, and fuzzy control. For further study, we will attend to the algebraic structure of $F P-\mathcal{C M F} \mathcal{S E S}$ such as group, ring and field. We plan to extend this concept to other types of algebraic structures such as monomial algebras [32] and semigroups $[33,34]$ in the future. We are eager to extend our work to other multi-criteria decision-making models and applications for modeling vagueness and uncertainty. 
Author Contributions: Y.A.-Q., M.H. and N.H. conceived and worked together to achieve this work.

Funding: This research was funded by Universiti Putra Malaysia under vote number 9001103.

Acknowledgments: We are indebted to Universiti Kebangsaan Malaysia for providing technical support and facilities for this research.

Conflicts of Interest: The authors declare that they have no conflict of interest.

\section{References}

1. Zadeh, L.A. Fuzzy sets. Inf. Control 1965, 8, 338-353. [CrossRef]

2. Atanassov, K.T. Intuitionistic fuzzy sets. Fuzzy Sets Syst. 1986, 20, 87-96. [CrossRef]

3. Sebastian, S.; Ramakrishnan, T.V. Multi-fuzzy sets. Int. Math. Forum 2010, 5, 2471-2476.

4. Sebastian, S.; Ramakrishnan, T.V. Multi-fuzzy sets: An extension of fuzzy sets. Fuzzy Inf. Eng. 2011, 3, 35-43. [CrossRef]

5. Lukovac, V.; Popović, M. Fuzzy Delphi approach to defining a cycle for assessing the performance of military drivers. Decis. Mak. Appl. Manag. Eng. 2018, 1, 67-81. [CrossRef]

6. Stanujkić, D.; Karabašević, D. An extension of the WASPAS method for decision-making problems with intuitionistic fuzzy numbers: a case of website evaluation. Oper. Res. Eng. Sci. Theory Appl. 2018, 1, 29-39. [CrossRef]

7. Molodtsov, D. Soft set theory-First results. Comput. Math. Appl. 1999, 37, 19-31. [CrossRef]

8. Maji, P.K.; Biswas, R.; Roy, A.R. Fuzzy soft set theory. J. Fuzzy Math. 2001, 9, 589-602.

9. Alcantud, J.; Muñoz Torrecillas, M. Intertemporal Choice of Fuzzy Soft Sets. Symmetry 2018, 10, 371. [CrossRef]

10. Maji, P.K.; Biswas, R.; Roy, A.R. Intuitionistic fuzzy soft sets. J. Fuzzy Math. 2001, 9, 677-692.

11. Garg, H.; Arora, R. Generalized intuitionistic fuzzy soft power aggregation operator based on t-norm and their application in multi criteria decision-making. Int. J. Intell. Syst. 2018, 34, 215-246. [CrossRef]

12. Yang, Y.; Tan, X.; Meng, C. The multi-fuzzy soft set and its application in decision making. Appl. Math. Model. 2013, 37, 4915-4923. [CrossRef]

13. Alkhazaleh, S.; Salleh, A.R. Soft expert sets. Adv. Decis. Sci. 2011, 2011, 757868. [CrossRef]

14. Alkhazaleh, S.; Salleh, A.R. Fuzzy soft expert set and its application. Appl. Math. 2014, 5, 1349-1368. [CrossRef]

15. Al-Qudah, Y.; Hassan, N. Bipolar fuzzy soft expert set and its application in decision making. Int. J. Decis. Sci. 2017, 10, 175-191. [CrossRef]

16. Adam, F.; Hassan, N. Multi Q-fuzzy parameterized soft set and its application. J. Intell. Fuzzy Syst. 2014, 27, 419-424.

17. Uluçay, V.; Şahin, M.; Hassan, N. Generalized neutrosophic soft expert set for multiple-criteria decision-making. Symmetry 2018, 10, 437. [CrossRef]

18. Abu Qamar, M.; Hassan, N. Generalized Q-neutrosophic soft expert set for decision under uncertainty. Symmetry 2018, 10, 621. [CrossRef]

19. Ramot, D.; Milo, R.; Friedman, M.; Kandel, A. Complex fuzzy sets. IEEE Trans Fuzzy Syst. 2002, 10, 171-186. [CrossRef]

20. Ramot, D.; Friedman, M.; Langholz, G.; Kandel, A. Complex fuzzy logic. IEEE Trans Fuzzy Syst. 2003, 11, 450-461. [CrossRef]

21. Singh, P.K. Granular-based decomposition of complex fuzzy context and its analysis. Prog. Artif. Intell. 2019, 1-13. [CrossRef]

22. Singh, P.K. Bipolar $\delta$-equal complex fuzzy concept lattice with its application. Neural Comput. Appl. 2019, 1-18. [CrossRef]

23. Al-Qudah, Y.; Hassan, N. Operations on complex multi-fuzzy sets. J. Intell. Fuzzy Syst. 2017, 33, 1527-1540. [CrossRef]

24. Al-Qudah, Y.; Hassan, N. Complex multi-fuzzy soft set: Its entropy and similarity measure. IEEE Access 2018, 6, 65002-65017. [CrossRef]

25. Al-Qudah, Y.; Hassan, N. Complex multi-fuzzy soft expert set and its application. Int. J. Math. Comput. Sci. 2019, 14, 149-176. 
26. Çă̆man, N.; Çitak, F.; Enginoğlu, S. FP-soft set theory and its applications. Ann. Fuzzy Math. Inform. 2011, 2, 219-226.

27. Çă̆man, N.; Çitak, F.; Enginoğlu, S. Fuzzy parameterised fuzzy soft set theory and its applications. Turk. J. Fuzzy Syst. 2010, 1, 21-35.

28. Deli, I.; Cagman, N. Intuitionistic fuzzy parameterized soft set theory and its decision making. Appl. Soft Comput. 2015, 28, 109-113. [CrossRef]

29. Deli, I.; Karatas, S. Interval valued intuitionistic fuzzy parameterized soft set theory and its decision making. J. Intell. Fuzzy. Syst. 2016, 30, 2073-2082. [CrossRef]

30. Bashir, M.; Salleh, A.R. Fuzzy parameterized soft expert set. Abstr. Appl. Anal. 2012, 2012, 258361. [CrossRef]

31. Hazaymeh, A.; Abdullah, I.B.; Balkhi, Z.; Ibrahim, R. Fuzzy parameterized fuzzy soft expert set. Appl. Math. Sci. 2012, 6, 5547-5564.

32. Belov, A.Y.; Borisenko, V.V.; Latyshev, V.N. Monomial algebras. J. Math. Sci. 1997, 87, 3463-3575. [CrossRef]

33. Belov, A.Y.; Ivanov, I.A. Construction of semigroups with some exotic properties. Acta Appl. Math. 2005, 85, 49-56. [CrossRef]

34. Ivanov-Pogodaev, I.; Malev, S.; Sapir, O. A construction of a finitely presented semigroup containing an infinite square-free ideal with zero multiplication. Int. J. Algebra Comput. 2018, 28, 1565-1573. [CrossRef]

(C) 2019 by the authors. Licensee MDPI, Basel, Switzerland. This article is an open access article distributed under the terms and conditions of the Creative Commons Attribution (CC BY) license (http:/ / creativecommons.org/licenses/by/4.0/). 